\title{
Constant acceleration exit of two-dimensional free-surface-piercing bodies
}

\author{
Rasadurai Rajavaheinthan, Martin Greenhow* \\ Brunel University, Uxbridge UB8 3PH, United Kingdom
}

\begin{abstract}
The forced constant acceleration exit of two-dimensional bodies through a free-surface is computed for various $2 \mathrm{D}$ bodies (symmetric wedges, asymmetric wedges, truncated wedges and boxes). The calculations are based on the fully non-linear time-stepping complex-variable method of Vinje and Brevig (1981). The model was formulated as an initial boundary-value problem (IBVP) with boundary conditions specified on the boundaries (dynamic and kinematic free-surface boundary conditions) and initial conditions at time zero (initial velocity and position of the body and free-surface particles). The formulated problem was solved by means of a boundary-element method using collocation points on the boundary of the domain and stepped forward in time using Runge-Kutta and Hamming predictor-corrector methods. Numerical results for the deformed free-surface profile, pressure along the wetted region of the bodies and force experienced by the bodies are given for the exit. The analytical added-mass force is presented for the exit of symmetric wedges and boxes with constant acceleration using conformal mappings. To verify the numerical results, the added-mass force and the numerical force are compared and give good agreement for the exit of a symmetric wedge at a time zero $(t=0)$ as expected but only moderate agreement for the box.
\end{abstract}

Keywords: Water exit, Nonlinear free surface, Complex potential, Conformal mapping, Added-mass force, IBVP

\section{Introduction}

The study of water entry has a wide range of applications in ocean engineering, especially in extreme or survival situations such as ship slamming or the ditching of aircraft. For such situations it is vital to know the pressure distribution on the submerged part of the body and the force acting on it. Because of the nature of the free-surface conditions, the flow is, in general, difficult to calculate. However, for the above applications, at least in the early stages of entry, the flow can be realistically treated as potential and only in the vertical plane so that the problem is twodimensional. Moreover, for high-speed entry gravity is usually ignored. In his seminal paper Wagner [16] also assumed that the deadrise angle was small, allowing the matching of an inner flow around an expanding flat plate to be matched to a planing-plate flow representing the spray jet. For a modern treatment see [7]. Another approach for wedges moving with constant speed (more generally with displacement being some power of time $t)$ is to exploit self-similarity, see $[5,17]$.

*Corresponding author. Tel: +4401895 265622.

Email address: Martin.Greenhow@brunel.ac.uk
In this paper we focus our attention on the study of water exit of surface-piercing bodies. Although this problem is usually less violent than that of entry, it has received far less attention in the literature. Perhaps part of the reason here is that none of the above approximations are valid. The initial draft of the body introduces a length scale which precludes self-similarity; equally gravity is essential to the filling of the hole that would otherwise be left by the exiting body. These two constraints imply that the problem is essentially Froude-number dependent and this makes analytical progress virtually impossible. This does not explain the dearth of published experimental results though; perhaps the exit problem is considered less important in engineering applications. Such a view is not valid for ship slamming, where velocity of entry of the ship's bow is determined by the previous motion during the exit phase. Another application is in marine crane operations where one needs to keep control of a body being lowered through the free surface in wave conditions to avoid snatching of the cables. This involves exit as well as entry relative to the moving wave surface. 
State-of-the-art engineering practice is reviewed in DNV [4]. We exploit the complex potential method of Longuet-Higgins and Cokelet [9] for extreme waves and further developed by Vinje and Brevig [14,15] for floating or submerged bodies. Greenhow [6] used this method to study the exit of submerged cylinders and presented initial results for wedge exit. We here present interesting results for the exit of symmetric wedges, asymmetric wedges, truncated wedges and a box body. The acceleration of the exiting body is constant and comparable to gravity, meaning that the effect of gravity on the loads will be significant. Certainly some of these bodies are likely to shed strong vortices from their corners, but these are ignored in this study. We hope that the present results will act as benchmarks and stimulate further theoretical and experimental work to assess the effects of vortices.

\section{Mathematical methods}

We can describe the motion of the system of fluid particles and the moving body in a two-dimensional complex Cartesian coordinate system. The particles on the body surface and the free surface can be considered by a mixed Eulerian and Lagrangian description, respectively. We assume the fluid is incompressible and the flow around the body irrotational so that the potential flow theory is applicable. The mathematical formulation and the solution of Vinje and Brevig's numerical technique are explained in the Appendices $\mathrm{A}$ and $\mathrm{B}$ for the convenience of the reader (especially given the age and relative obscurity of their publications).

The method is a boundary-integral method based on Cauchy's theorem, where the contour $C$ comprises the free surface, the body surface, the bottom and distant vertical boundaries (see Fig. A.16). We let a collocation point $z_{k}$ move onto the boundary from outside $C$ giving

$$
\pi \psi\left(z_{k}\right)+\operatorname{Re}\left\{\int_{C_{0}} \frac{\phi+i \psi}{z-z_{k}} d z\right\}=0, \quad \forall z_{k} \in C^{\phi},
$$

or

$$
\pi \phi\left(z_{k}\right)+\operatorname{Re}\left\{i \int_{C_{0}} \frac{\phi+i \psi}{z-z_{k}} d z\right\}=0, \quad \forall z_{k} \in C^{\psi},
$$

where $C^{\phi}$ is the part of $C$ where the velocity potential $\phi$ is known, $C^{\psi}$ that where the stream function $\psi$ is known and $C_{0}$ is $C$ minus a semi-circular indent into the fluid at $z_{k}$. As discussed by Greenhow [7] the intersection points of the body and free surface require special treatment. Here both $\phi$ and $\psi$ are known from the boundary conditions and so do not require calculation from Eq. (1) or Eq. (2). To step forwards in time these points are treated as ordinary free-surface particles and their new positions are found, then corrected by moving them horizontally back onto the body surface. Greenhow [6] showed that this essentially pragmatic approach leads to sensible results that respect the criterion of arc length constancy for zero gravity wedge entry (a consequence of self-similarity). However, it is not known if the predicted negative pressures that occur near the jet tip are physical. These also occur near the intersection points for wedge exit, see Figs. 5(b) and 8(b), especially at $\tau=0$, where the free-surface calculations presumably do not allow sufficiently rapid ventilation down the wedge surface from the free surface. Note that the predicted negative pressures that occur near sharp corners, see Fig. 12(c), arises not from the treatment of the intersection points, but rather reflect the inability of the present inviscid model to cope with the flow singularity at sharp corners; in reality vortices will be shed here. However, these low pressure regions do not significantly affect global loads on the body in most of the cases considered here.

\section{Results and discussions}

In this section, we present a set of selected results for water exit of the two-dimensional bodies shown in Fig. 1 exiting with constant acceleration. Results for an extensive range of parameters given in Fig. 1 are documented in Rajavaheinthan [12]. This paper presents results for the symmetric wedge SW30 of half angle $30^{\circ}$, the asymmetric wedges AW1 of left angle $\beta_{h}=0^{\circ}$ and right angle $\alpha_{h}=30^{\circ}$ and AW2 of left angle $\beta_{h}=-20^{\circ}$ and right angle $\alpha_{h}=30^{\circ}$, the truncated wedge TW30 of nondimensional bottom width $\widehat{b}=0.6$ and left and right angle of $30^{\circ}$ and the box BX1 of nondimensional bottom-half width $\widehat{b}=0.67$. The bodies are submerged initially at a nondimensional depth of -1 and exiting with a nondimensional constant acceleration $G_{\tau}$ of 1 , see Eqs. (5) and (8). To verify the computed results, we carry out a set of tests such as convergence checks, time effect on the fluid and body motion and the added mass effect on the force for the symmetric wedges and boxes.

We give computed results for the physical quantities (free-surface elevation, pressure distribution and force) in non-dimensional form. 


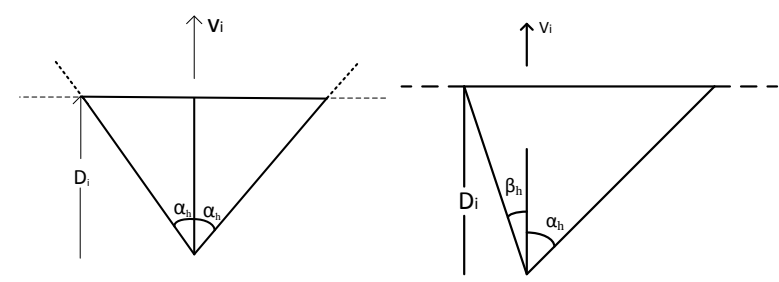

(a) Symmetric wedge

(b) Asymmetric wedge
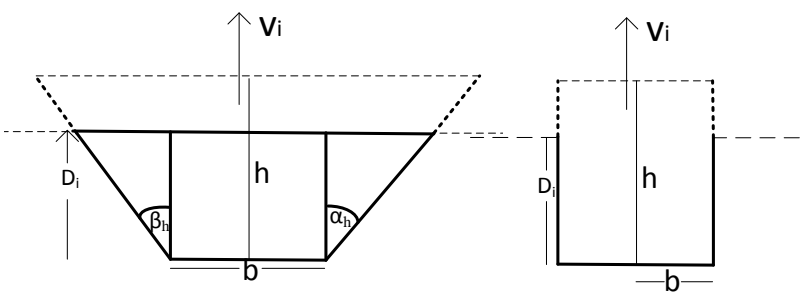

(c) Truncated wedge

(d) Box

Figure 1: Geometrical representation of the different shaped bodies for water exit cases.

The dimensional characteristic time $C_{t}$ is defined by

$$
C_{t}=\sqrt{\frac{D_{i}}{g}},
$$

where $D_{i}$ is the initial submergence depth of the body below the free surface at rest and gravitational field is denoted by $g$.

The dimensionless time $\tau$ is given by dividing time $t$ by the characteristic time $C_{t}$ as follows

$$
\tau=\frac{t}{C_{t}}
$$

Spatial coordinates are made dimensionless by dividing by the initial submergence depth $D_{i}$ giving

$$
\widehat{z}=\frac{z}{D_{i}}=\frac{x+i y}{D_{i}}=D_{x}+i D_{y} .
$$

The total pressure (static and dynamic) $P$ computed by the Bernoulli's equation is divided by the initial hydrostatic pressure at $y=-D_{i}$ to get the non-dimensional pressure $\widehat{P}$ as below

$$
\widehat{P}=\frac{P}{D_{i} \rho_{w} g}
$$

where $\rho_{w}$ is density of water. The vertical force $F$ experienced by the body is computed using the fully nonlinear numerical method which is non-dimensionalised by dividing the force by the initial buoyancy force and can be expressed as

$$
\widehat{F}=\frac{F}{\rho_{w} g \forall},
$$

where $\forall$ is the initial submerged volume (per unit length of the wedge prism). The dimensionless acceleration parameter can be defined by dividing the original acceleration of the body $a$ by the gravity $g$ as

$$
G_{\tau}=\frac{a}{g}
$$

In this paper the nondimensional water depth is large. Extensive calculations for both entry and exit cases presented by Rajavaheinthan [12] show that there are no significant depth effects until the nondimensional water depth is less than 2. This will be reported on in a subsequent paper.

\subsection{Convergence check}

To check convergence of the numerical results, we consider the wedge SW30 exiting with constant acceleration. For the wedge, the free-surface profile and pressure distribution are computed for different non-dimensional time step sizes $D \tau$ with all other variables being the same. The results for the exit of the wedge are plotted at a particular non-dimensional time $\tau$ for different non-dimensional time step size $D \tau$. For example, Fig. 2 shows the free-surface profile and pressure distribution computed for the wedge SW30 exiting with nondimensional acceleration $G_{\tau}=0.8$ which is plotted for different non-dimensional time step size $D \tau$ of $0.1,0.01$ and 0.001 at a non-dimensional time $\tau=1$.

We see in Fig. 2 that the results show a good agreement in this convergence test. Results for the free surface and pressure at different time step sizes coincide for different time step sizes. From these convergence results, we use the non-dimensional time step size of 0.1 to do the most of the calculations of all other shapes exiting with constant acceleration. As we increase non-dimensional time step size from 0.001 to 0.1 , we can see only a small deviation in the results.

Another aspect of convergence is the spatial distance between free-surface points and body points. Initially the Lagrangian free-surface points are not spaced equally, but in geometric ratio, $R_{f}$, getting closer as one approaches the intersection points. The body points (not Lagrangian but fixed to the body) are 
also in a similar geometric ratio, $R_{b}$, getting closer as one approaches the vertex. Both can be seen in Figs. 3(a) and 4(a) for $\tau=1$. It is important to make the free-surface and body point spacings near the intersection points roughly the same, but otherwise small variations in these spacing ratio or number of points chosen on free and body surfaces do not have a significant effect of the free surface profiles which are relatively flat for the accelerating cases discussed here. Barringer [2] carried out a series of test cases for constant velocity wedge entry and exit. For entry, self-similarity provides a stringent check on the time and space resolutions. For exit some of his cases showed wave-like motion causing jets to form at the intersection and hence numerical breakdown. He speculates that this may be partly physical, but needs to be suppressed by coarser resolution of the free surface to allow the calculations to proceed. In the present and more physical constant acceleration cases no such jets occur. Our calculations conform with Barringer's recommendations (see Figs. 3 and 4) and we use $R_{b}=0.98, R_{f}=0.85$, the number of body points $\mathrm{NBODY}=50$ and the number of free-surface points $\mathrm{NF}=70$ throughout for the wedges, whilst for the truncated wedge and the box, $R_{b}$ refers to the point spacing ratio from the initial free surface to the corner, with equal spacing along the flat bottom surfaces. This ensures that with the recommended non-dimensional time step of 0.1 , the free-surface points move no more that $10 \%$ of the distance between points. Note that the modest number of free-surface points needed arises because, away from the body, the flow is essentially quiescent.

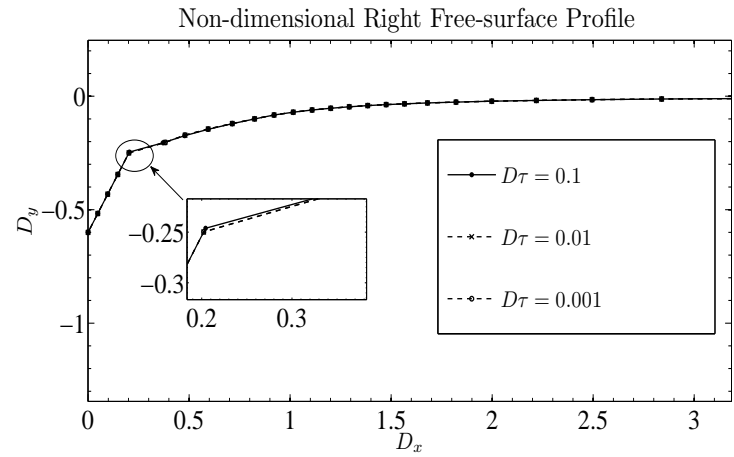

(a)

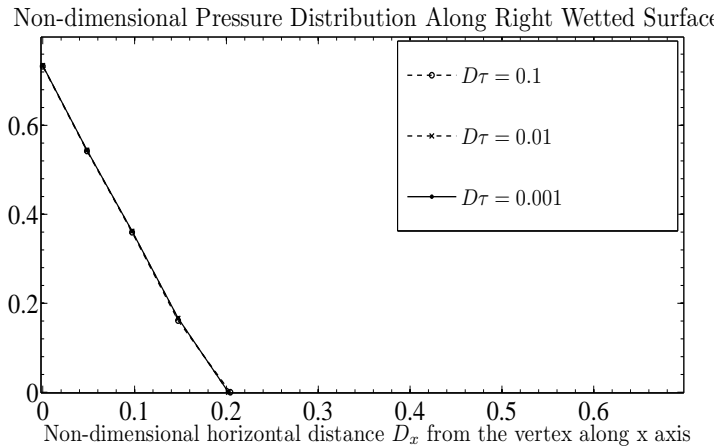

(b)

Figure 2: Convergence of the symmetric wedge SW30 submerged at a non-dimensional initial depth $\widehat{D}_{i}=-1$ exiting with constant acceleration of $G_{\tau}=0.8$ and plotted at a non-dimensional time $\tau=1$. $\alpha_{h}=30^{\circ}$. 


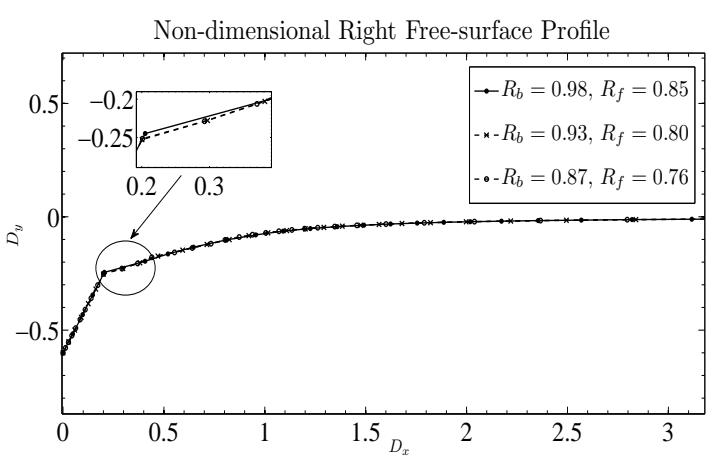

(a)

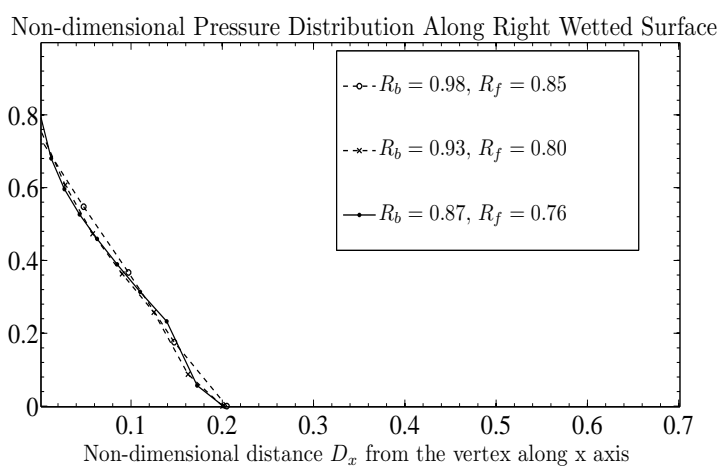

(b)

Figure 3: Convergence of the symmetric wedge SW30 submerged at a non-dimensional initial depth $\widehat{D}_{i}=-1$ exiting with constant acceleration of $G_{\tau}=0.8$ and plotted at a non-dimensional time $\tau=1$. $\alpha_{h}=30^{\circ} . R_{b}$ and $R_{f}$ are ratio of the body points and the free-surface point spacings, respectively; NBODY $=50$ and NF $=70$ are the number of points on the body surface and the free surface, respectively.

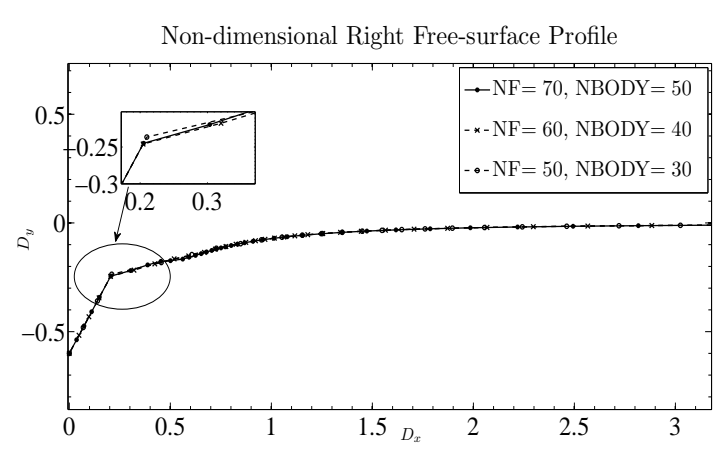

(a)

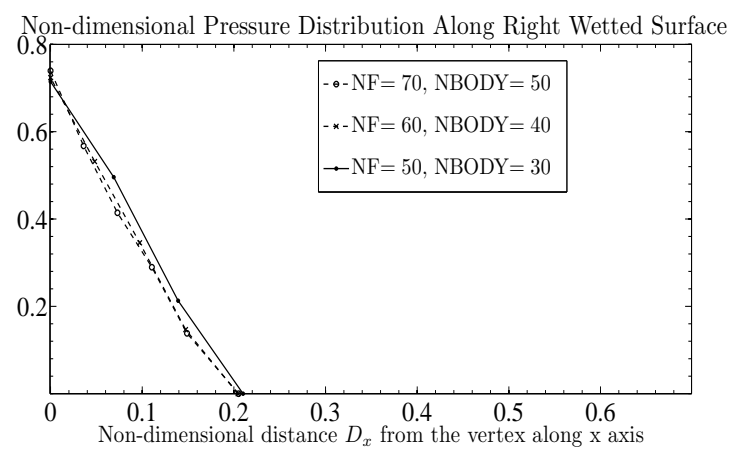

(b)

Figure 4: Convergence of the symmetric wedge SW30 submerged at a non-dimensional initial depth $\widehat{D}_{i}=-1$ exiting with constant acceleration of $G_{\tau}=0.8$ and plotted at a non-dimensional time $\tau=1$. $\alpha_{h}=30^{\circ}, R_{b}=0.98$ and $R_{f}=0.85$ are ratio of the body points and the free-surface point spacings, respectively; NBODY and NF are number of points on the body surface and the free-surface, respectively. 


\subsection{Time effect}

We compute the deformed free-surface profile, pressure along the wetted part of the body and the upward force experienced by the body using the non-linear theory. The computed results showing the time effect on free surface, pressure along the wetted surface of the bodies (see Fig. 1) and force at different stages of exit with constant acceleration are presented in this section. Fig. 5 shows the time effect of the symmetric wedge SW30 exiting with a nondimensional acceleration $G_{\tau}$ of 1. As for the symmetric wedge, Figs. 6 and 7 show the time effect of the asymmetric wedges AW2 and AW1 exiting water with a constant acceleration $G_{\tau}$ of 0.6 and 1 , respectively. Such a flow may be pertinent to the exit of propeller blades for a ship in ballast. Fig. 8 shows the computed results for the truncated wedge TW30 exiting water with a constant acceleration $\left(G_{\tau}\right)$ of 1 . Fig. 9 shows the computed results for the box body BX1 exiting water with a constant acceleration $G_{\tau}=0.5$.

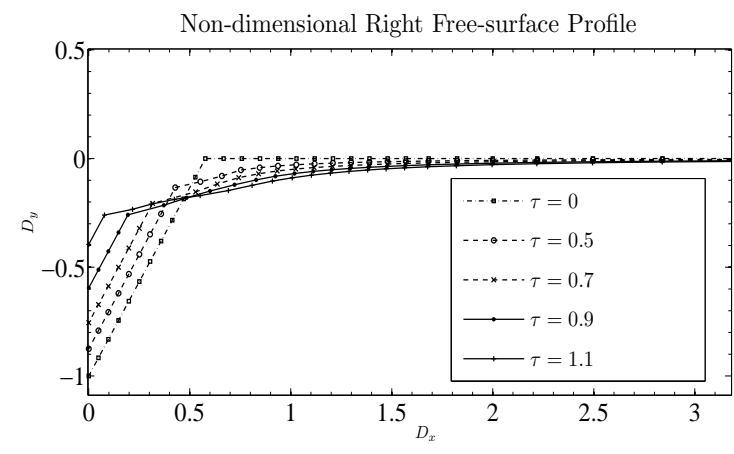

(a)

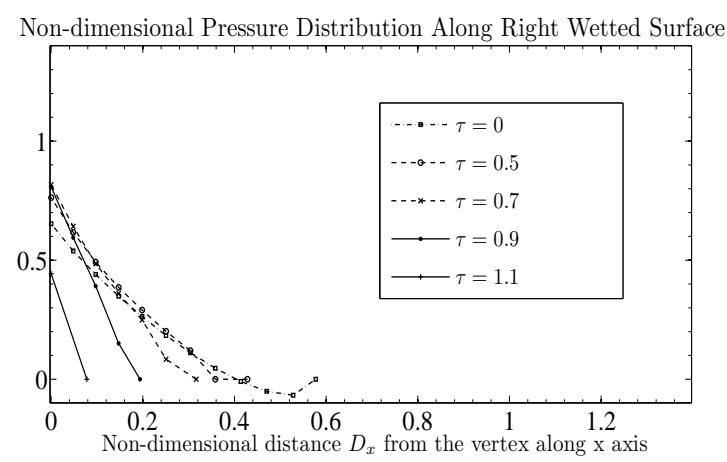

(b)

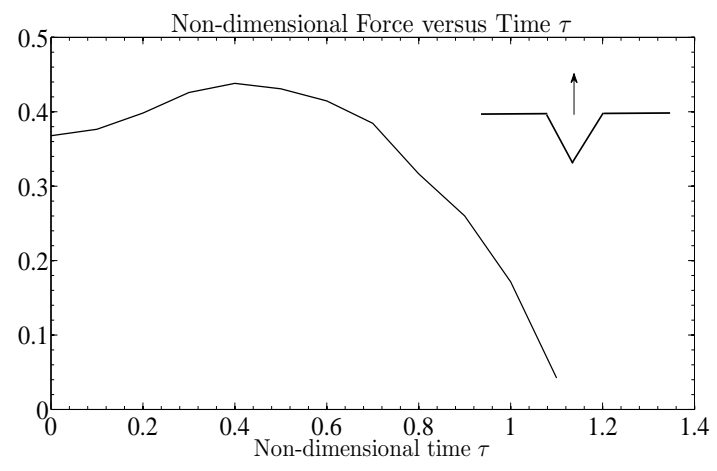

(c)

Figure 5: Time effect of the symmetric wedge SW30 submerged at a non-dimensional initial depth $\widehat{D}_{i}=-1$ exiting with constant acceleration $G_{\tau}=1: \alpha_{h}=30^{\circ}$. A cartoon showing the physical situation is given in the force graph (c), and in subsequent force graphs in this section. 


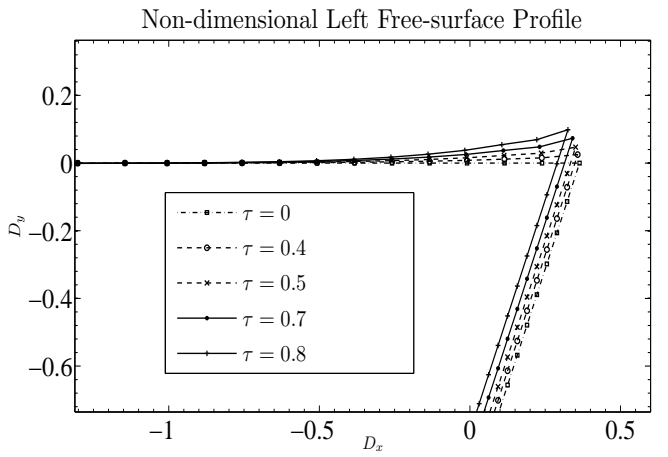

(a)

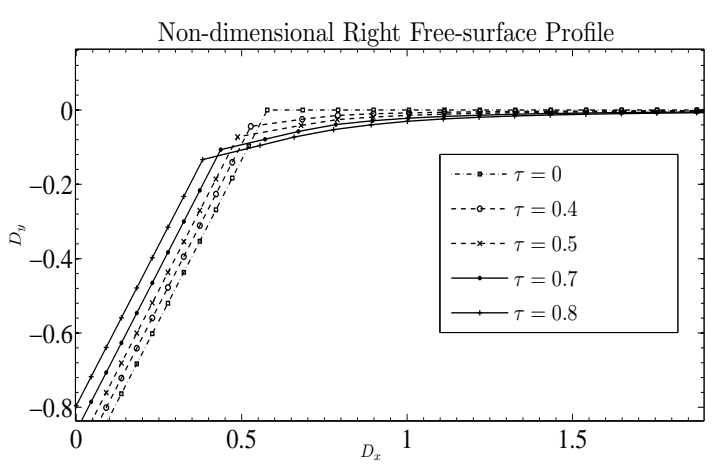

(b)

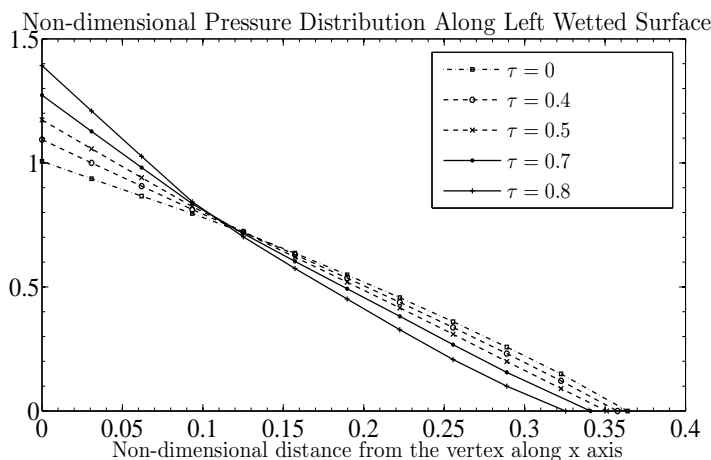

(c)

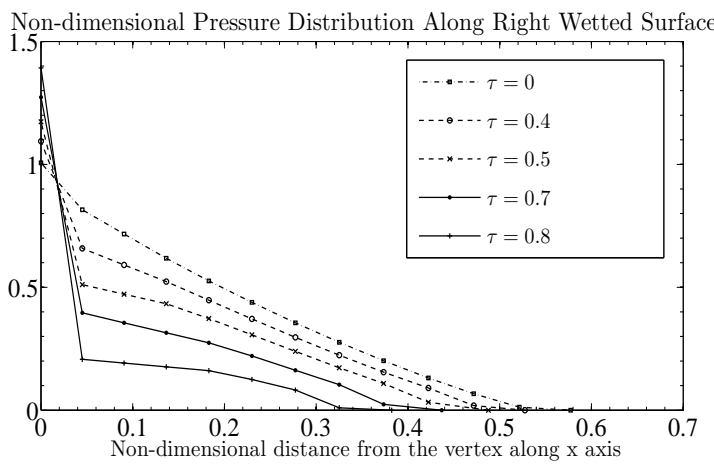

(d)

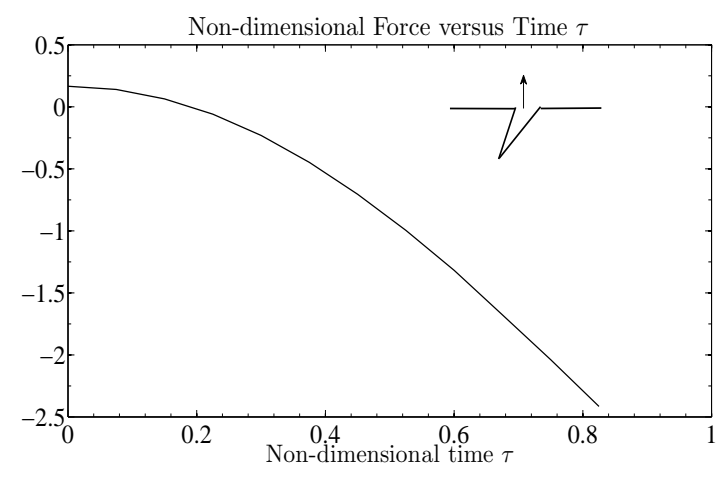

(e)

Figure 6: Time effect of the asymmetric wedge AW2 submerged at a non-dimensional initial depth $\widehat{D}_{i}=-1$ exiting with constant acceleration $G_{\tau}=0.6$ : $\alpha_{h}=30 \circ$ and $\beta_{h}=-20$ 。.

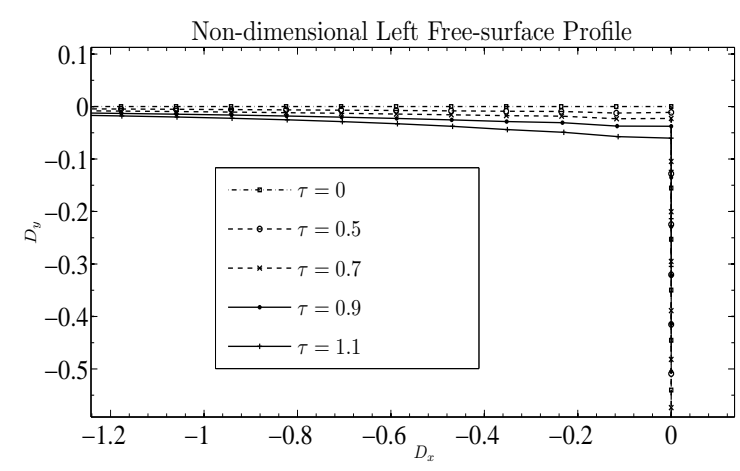

(a)

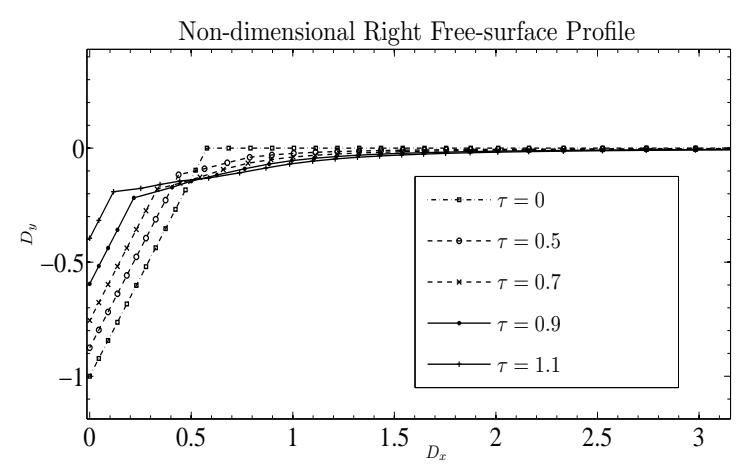

(b) 


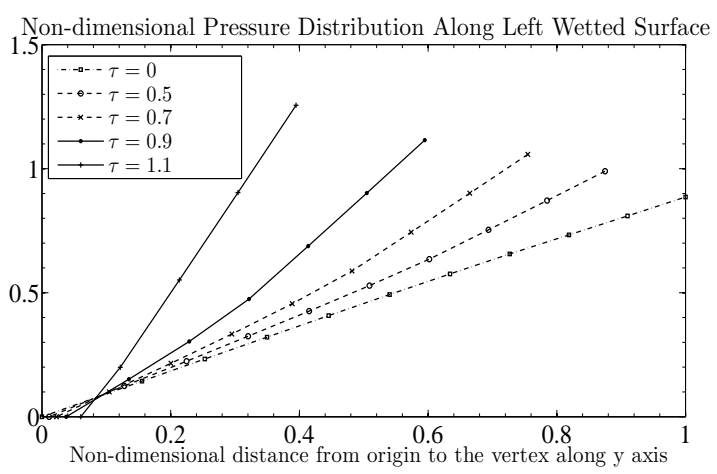

(c)

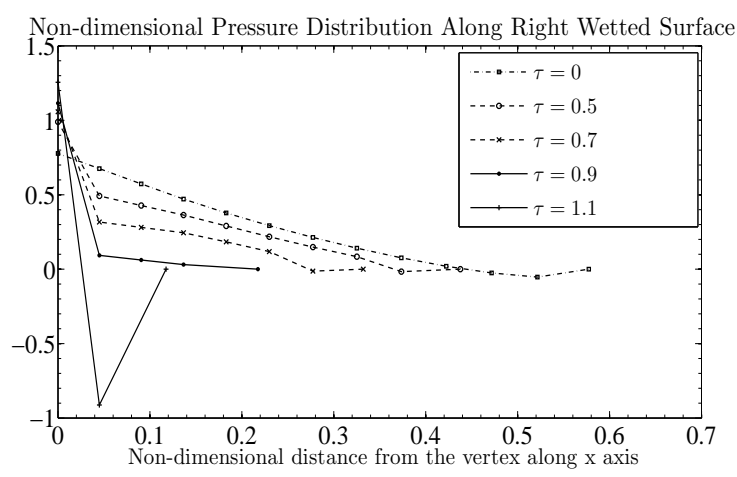

(d)

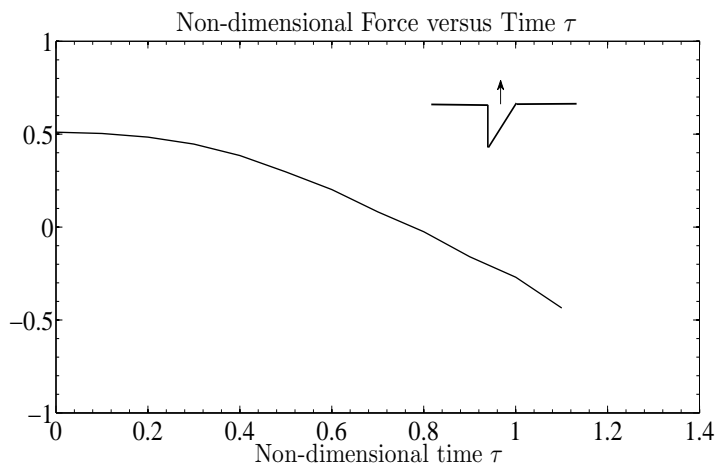

(e)

Figure 7: Time effect of the asymmetric wedge AW1 submerged at a non-dimensional initial depth $\widehat{D}_{i}=-1$ exiting with constant acceleration $G_{\tau}=1: \alpha_{h}=30^{\circ}$ and $\beta_{h}=0^{\circ}$.

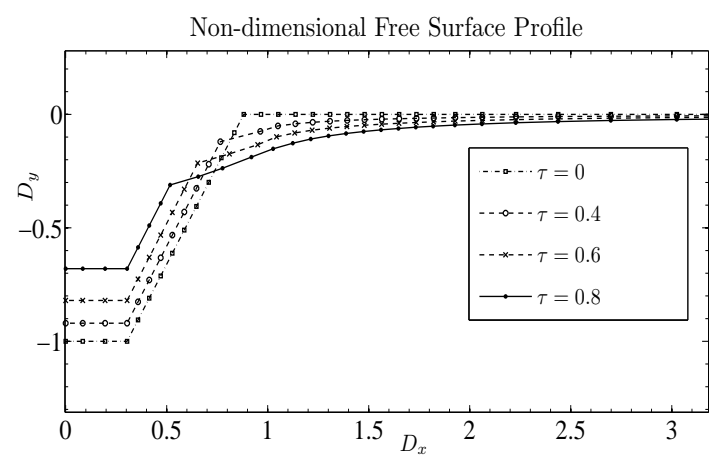

(a)

Non-dimensional Pressure Distribution Along Right Wetted Surface

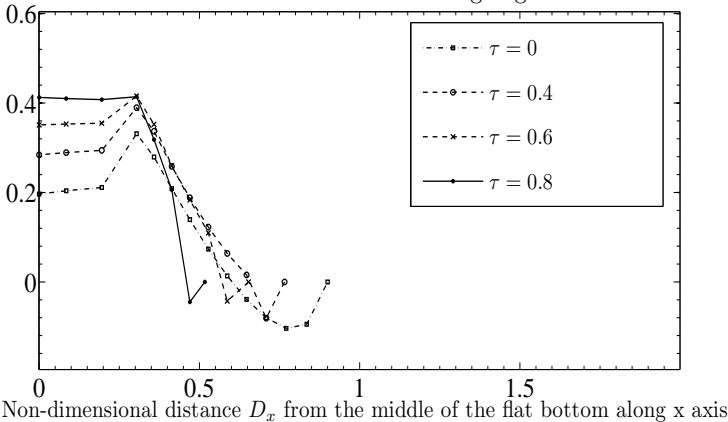

(b)

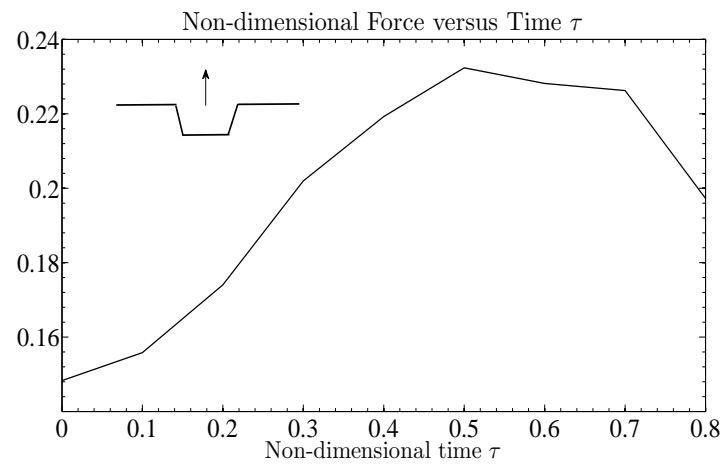

(c)

Figure 8: Time effect of the truncated wedge TW30 submerged at a non-dimensional initial depth $\widehat{D}_{i}=-1$ exiting with constant acceleration $G_{\tau}=0.5$ : nondimensional bottom width $\widehat{b}=0.6$. 


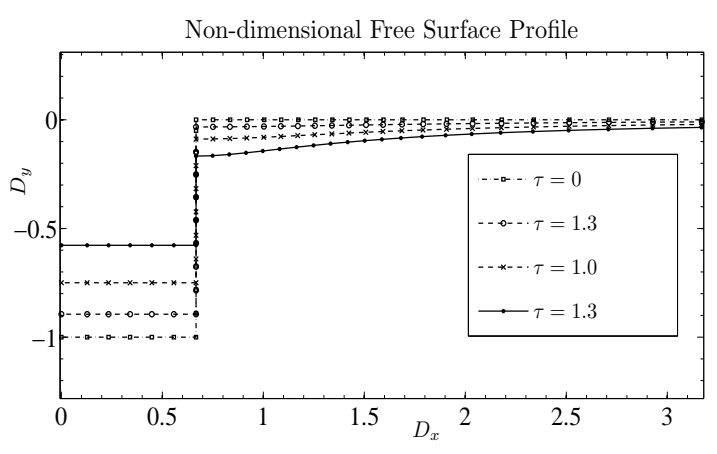

(a)

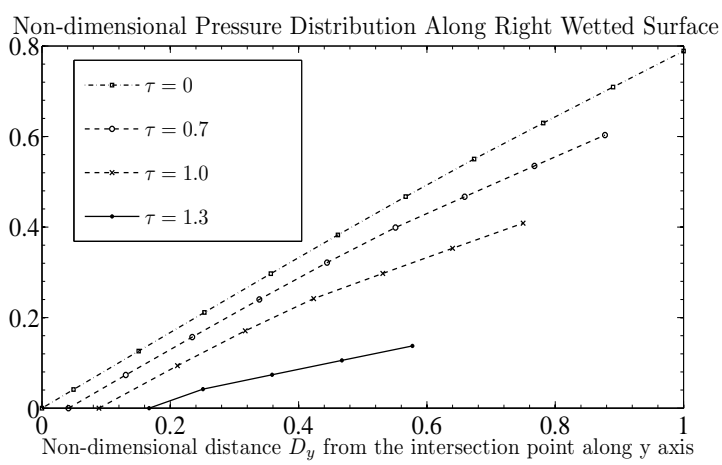

(b) along vertical surface

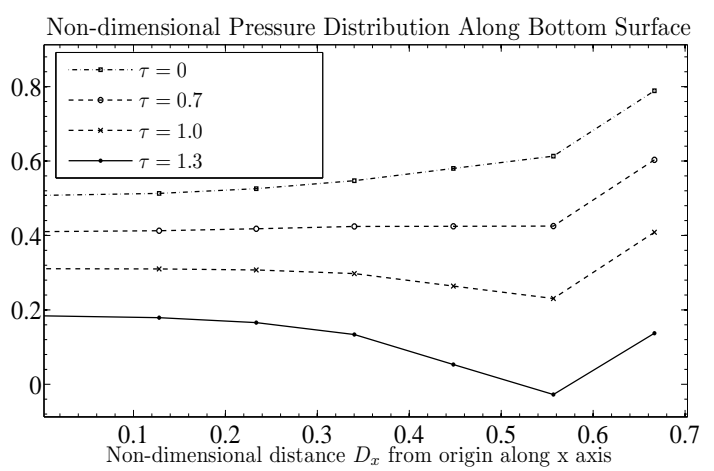

(c)

Despite the complexity of the flow, it is possible to make the following comments on these calculations. In all cases, the pressure and heave total force on the various bodies are far from the hydrostatic even at $\tau=0$. This arises from the $\frac{\partial \phi}{\partial t}$ term in Bernoulli's equation (A.4). The total upwards force in Fig. 5 increases initially; this is confirmed in Appendix $C$, where the simplified added-mass model includes a velocity square term that is always positive. Appendices $C$ and $D$ show that the added-mass model gives a reasonable description of the force difference (the total force minus the linearised hydrostatic force on that part of the body below $y=0$ rather than that on

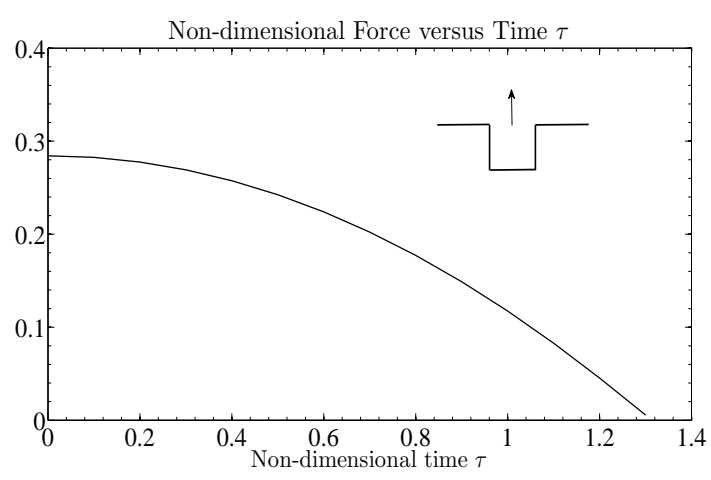

(d)

Figure 9: Time effect of the box BX1 submerged at a non-dimensional initial depth $\widehat{D}_{i}=-1$ exiting with constant acceleration $G_{\tau}=0.5$ : nondimensional bottom-half width $\widehat{b}=0.67$.

the wetted part of the body which is not known a priori).

For the asymmetric wedges AW2 and AW3 the pressures near the vertices need to be treated with caution; the velocity squared term $\omega \bar{\omega}$ of Bernoulli's equation (A.4) relies on the calculation of $\frac{d \beta}{d z}$ and this has an integrable singularity here. Consequently we cannot expect the pressures near the vertices or corners to converge as one increases the body point resolution here. It is therefore pertinent to examine how this might affect the free surface profiles and overall body forces. Tests at $\tau=0.5$ for AW1 and AW2 with NBODY varying from 30 to 60 show virtually indistinguishable free surface profiles in all cases (also for the box BX1 discussed below), and pressures on the wetted surfaces within about $2 \%$ except at the vertex where there the best resolution has pressures some $10 \%$ higher than the worst resolution calculations. We do not claim convergence at the vertex and in reality vortices will be shed here; nevertheless the overall vertical force is largely unaffected by the choice of body point resolution.

Note that for AW2, the force rapidly becomes negative because the pressure on the left surfaces pushes the body down, not up. Note also that the force on AW1 is not half that on SW30, i.e. the non-dimensional forces are different, because the flow is no longer symmetric. On the vertical left surface, the pressure has no effect on the upwards force. This force arises solely from the pressures in the right hand (inclined) surface, and these are affected by the flow asymmetry.

For the truncated wedge, the free surface is qualitatively similar to SW30. However, these calculations 
break down shortly after the last case shown when the intersection point will have to move around the knuckle and this is not possible using the current intersection point algorithm. Note also that the pressure near the knuckle becomes irregular again due to the singularity there, see Fig. 8(b). The same effect can be seen for the later stages of the box BX1, although negative pressures are avoided except at the bottom-surface point next to the corner at the end of the calculation when $\tau=1.3$, see Fig. 9(c). Varying the number of body points (NBODY $=30-50)$ shows no convergence in the pressure here or at the corner (as expected) but only modest agreement to within $20 \%$ for all points along the bottom surface. This may explain the divergence of the results for the force difference shown in Appendix D.

\subsection{Acceleration effect}

For each shape considered, to study the time effect of the constant acceleration exit, we present the computed free-surface profile, pressure distribution along the wetted part of the body and numerical total force experienced by the body as it exits through the free surface with different constant accelerations. The results are obtained by running the program with different constant accelerations while attaining the same distance travelled by the bodies. Figs. 10-14 show the acceleration effect of the symmetric wedge SW30, asymmetric wedges AW2 and AW1, truncated wedge TW30 and box BX1 exiting with different accelerations.

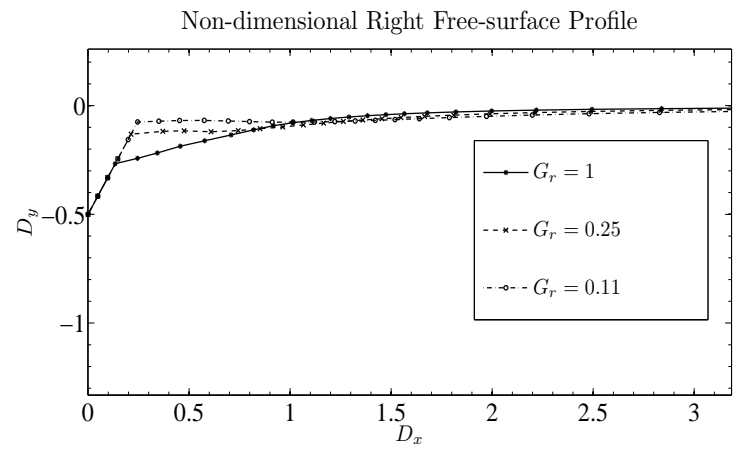

(a)

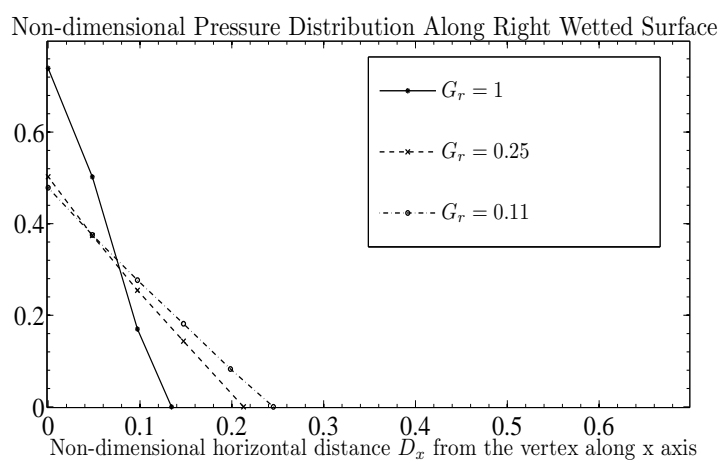

(b)

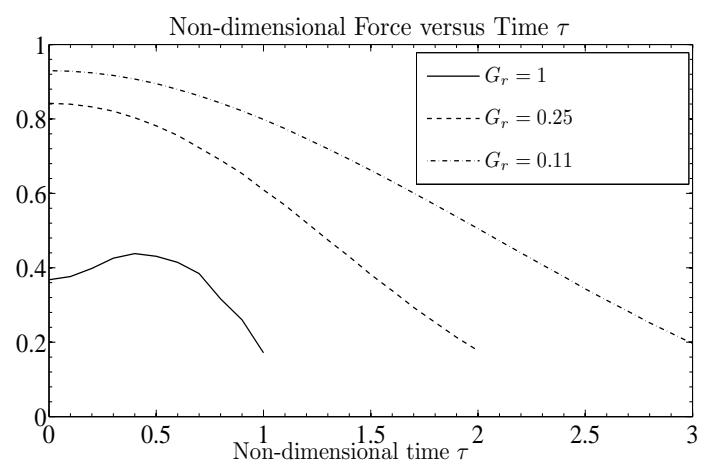

(c)

Figure 10: Acceleration effect of the symmetric wedge SW30 submerged at a non-dimensional initial depth $\widehat{D}_{i}=-1$ exiting with different constant accelerations $G_{\tau}: \alpha_{h}=30^{\circ}$. 


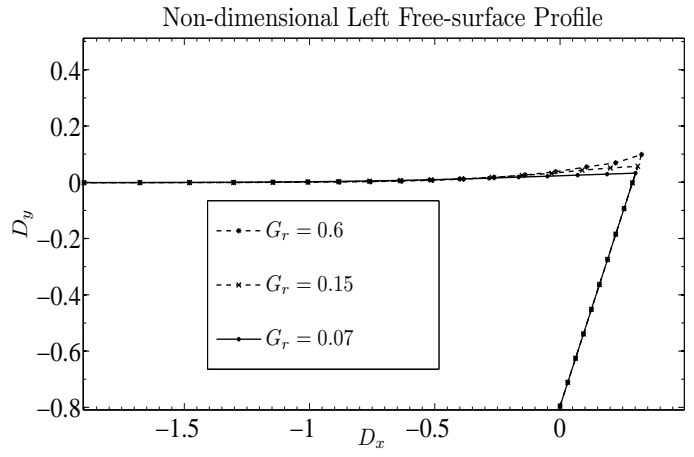

(a)

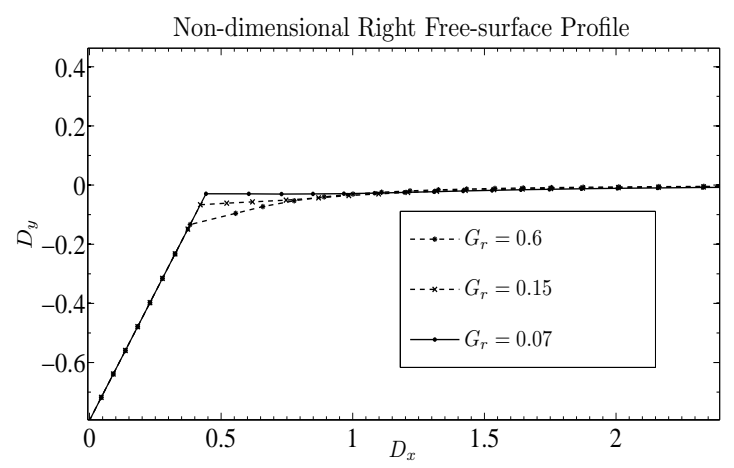

(b)

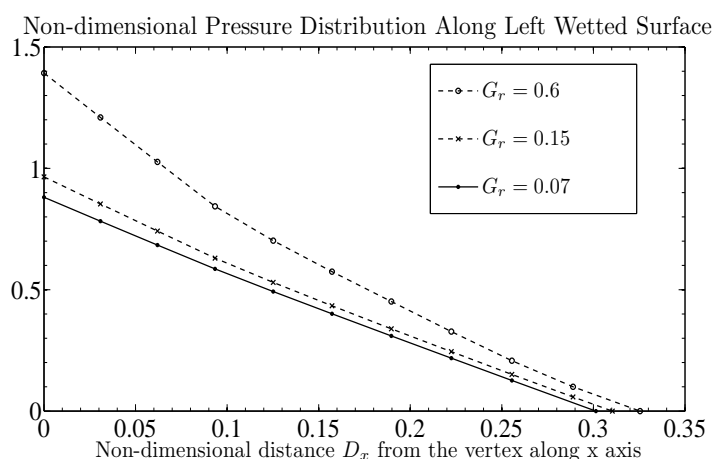

(c)

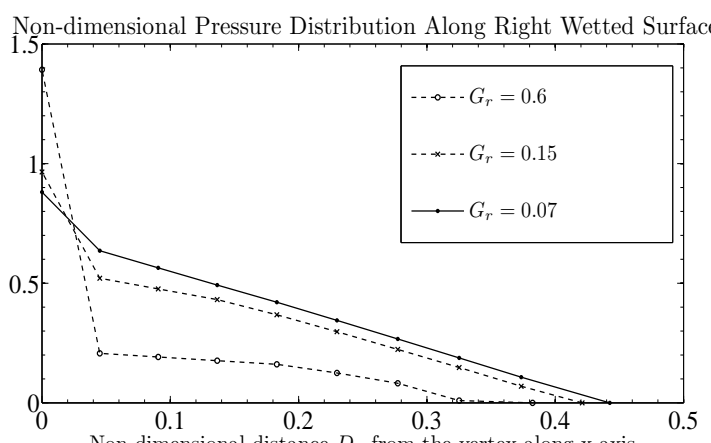

(d)

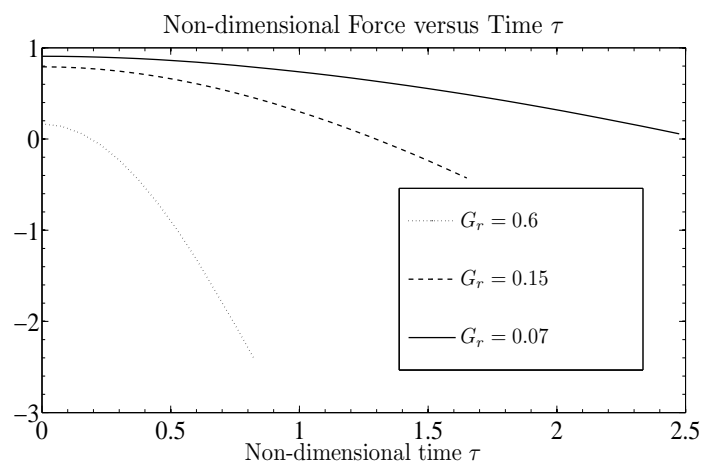

(e)

Figure 11: Acceleration effect of the asymmetric wedge AW2 submerged at a non-dimensional initial depth $\widehat{D}_{i}=-1$ exiting with different constant accelerations $G_{\tau}: \alpha_{h}=30^{\circ}$ and $\beta_{h}=-20^{\circ}$. 


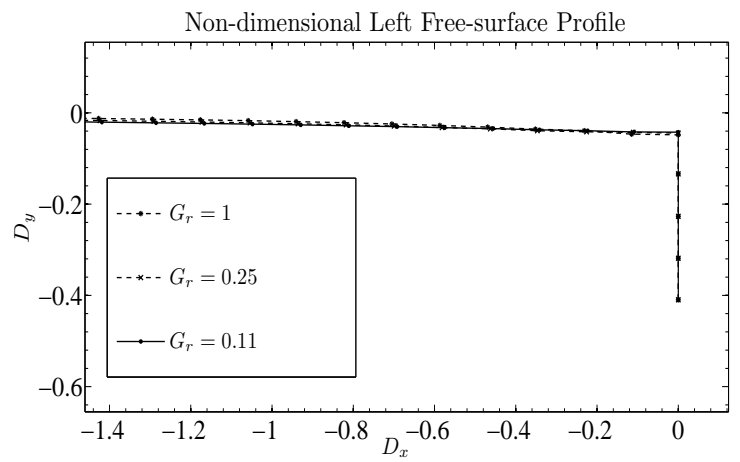

(a)

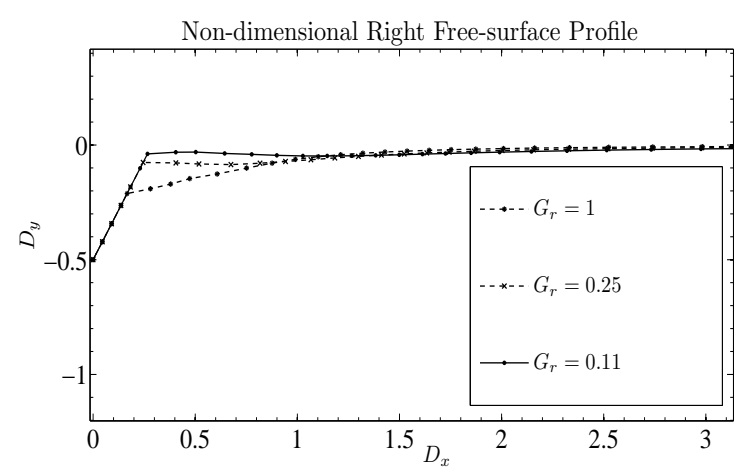

(b)

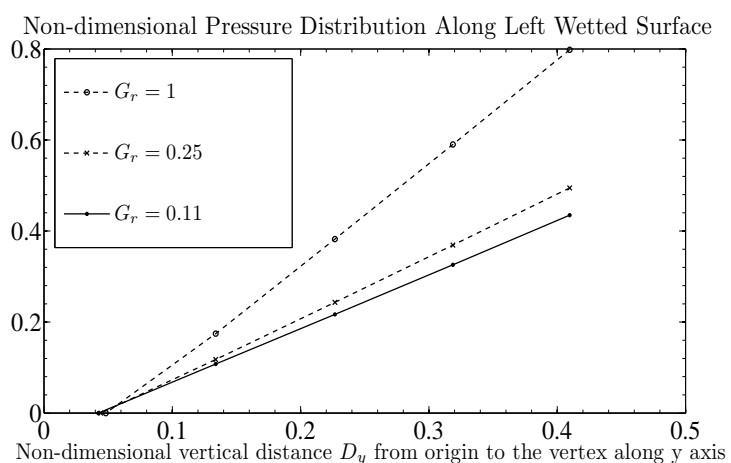

(c)

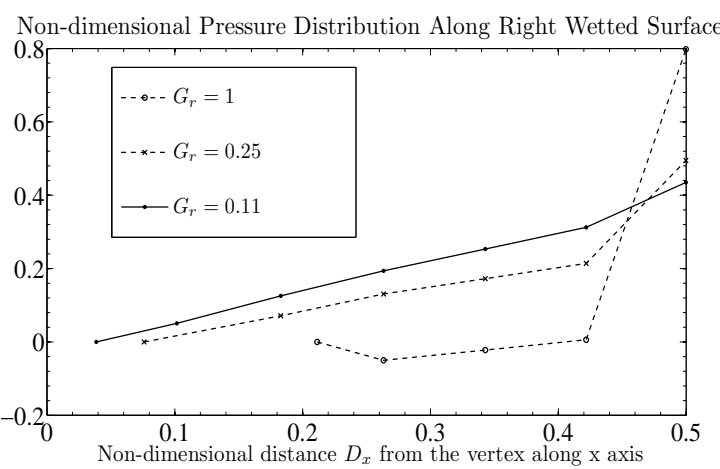

(d)

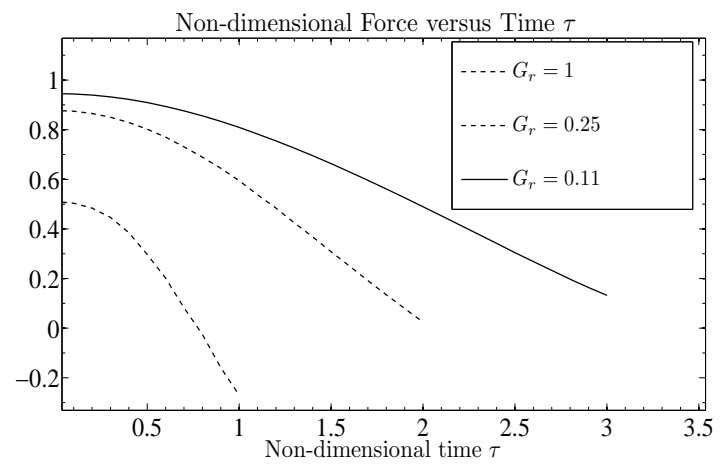

(e)

Figure 12: Acceleration effect of the asymmetric wedge AW1 submerged at a non-dimensional initial depth $\widehat{D}_{i}=-1$ exiting with different constant accelerations $G_{\tau}: \alpha_{h}=30^{\circ}$ and $\beta_{h}=0^{\circ}$. 


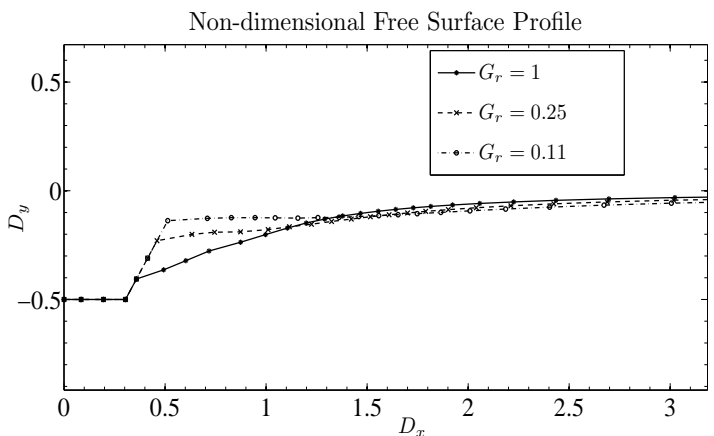

(a)

Non-dimensional Pressure Distribution Along Right Wetted Surface

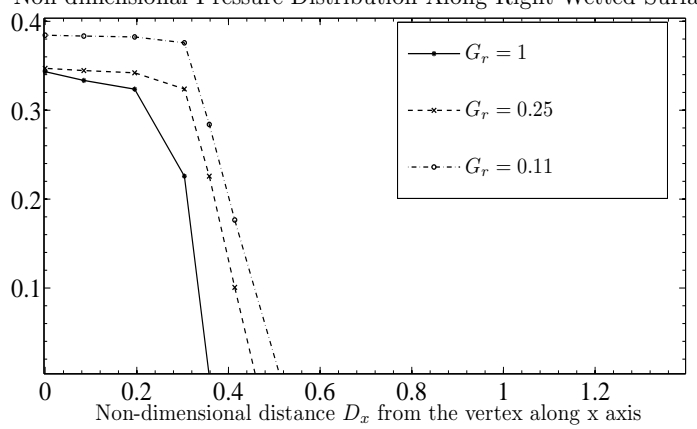

(b)

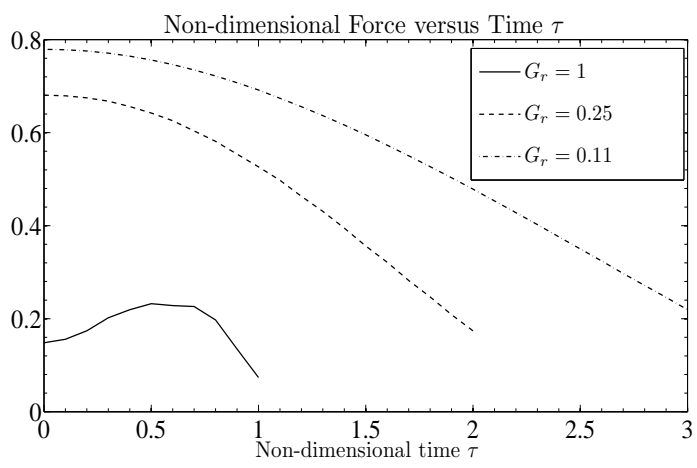

(c)

Figure 13: Acceleration effect of the truncated wedge TW30 submerged at a non-dimensional initial depth $\widehat{D}_{i}=-1$ exiting with different constant accelerations $G_{\tau}$ : nondimensional bottom width $\widehat{b}=$ 0.6 .

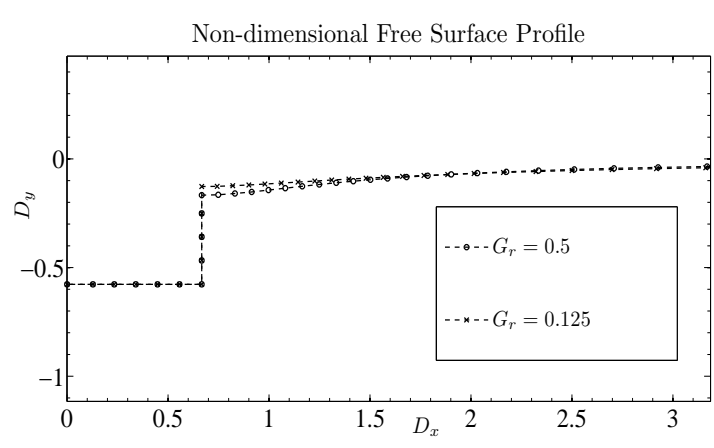

(a)

Non-dimensional Pressure Distribution Along Right Wetted Surface

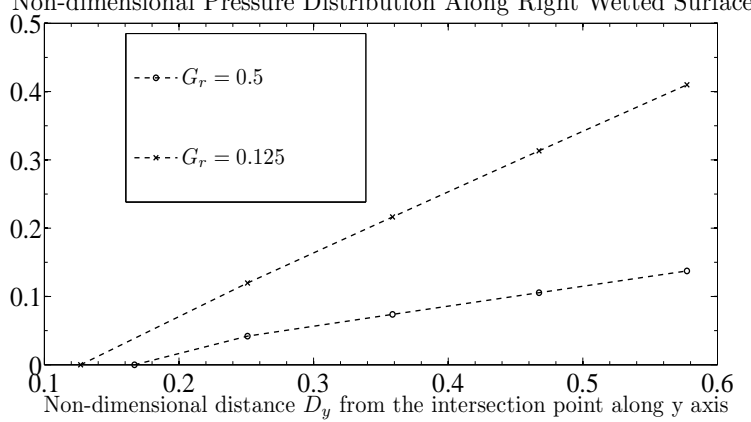

(b) along vertical surface

Non-dimensional Pressure Distribution Along Bottom Surface

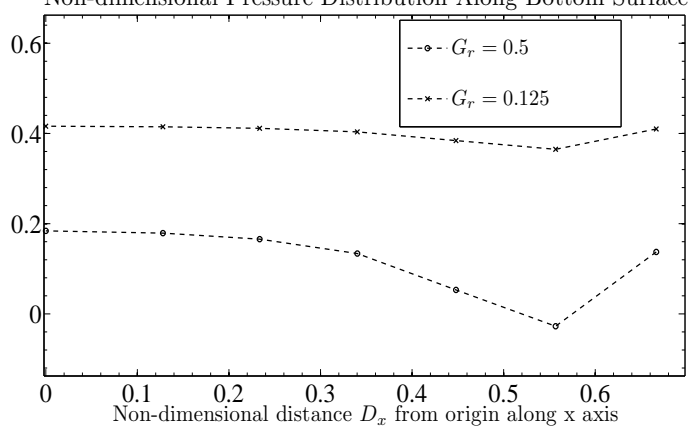

(c) 


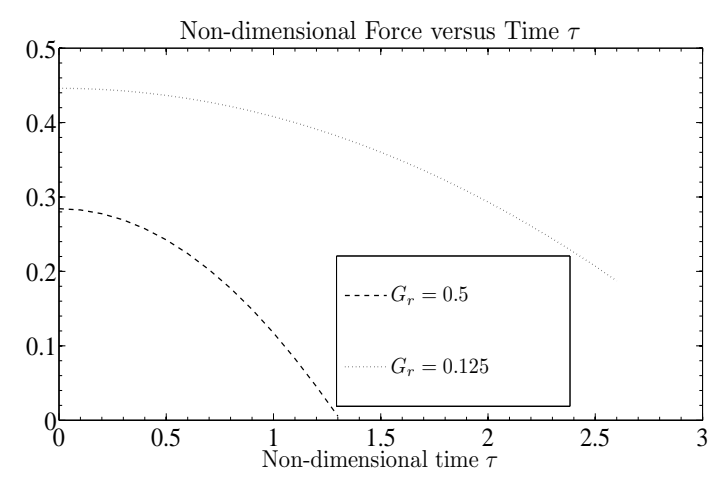

(d)

Figure 14: Acceleration effect of the box BX1 submerged at a nondimensional initial depth $\widehat{D}_{i}=-1$ exiting with different constant accelerations $G_{\tau}$ : nondimensional bottom-half width $\widehat{b}=0.67$.

In Rajavaheinthan [12] extensive results are also given for the constant velocity case. As pointed out by Korobkin ([18], private communication), this models an impulsively-started body motion, which is not entirely physical. In reality there must be some period of rapid acceleration, before the constant velocity phase which has zero acceleration. Referring to the right-hand side of Eq. (C.10) we see the somewhat surprising result that during this phase the force is always positive (i.e. upwards) for constant velocity since the $y$-derivative of the added mass is negative. Thus the body is effectively being pushed upwards by the fluid. Our common experience that it is difficult to accelerate bodies out of a fluid (i.e. negative hydrodynamic force) arises from the acceleration period only (according to this simplified model). To compare with experiments both phases of the motion would need to be calculated and hence the constant velocity cases are omitted from this paper.

Rajavaheinthan [12] also considers different wedge angles, but none have a deadrise less than $35^{\circ}$ because of the fast motion of the Lagrangian points at the free surface/body intersection. In contrast Korobkin [8] has attempted to model the exit of a low-deadrise angle wedge using a contracting flat plate, as in Wagner's model. This is not a fully-closed model and it is not yet clear how any numerical method could cope with this low-deadrise-angle regime to provide the contraction parameter needed by Korobkin's model. Even for the case SW30 presented here, with the deadrise angle of $60^{\circ}$, the negative pressures near the intersection points at $\tau=0$ shown in Fig. 5(b), cast doubt on the initial motion of the intersection points calculated here.
Rajavaheinthan [12] presents results for the speed of the intersection points for a symmetric wedge of half-angle of $30^{\circ}$ (as in SW30) during entry and exit at constant velocity. For entry/exit, the initial nondimensional speed is about $0.97 / 1.79$ respectively, and independent of the Froude number. We might expect this since the numerics are attempting to solve essentially the same problem for each Froude number i.e. that of flow around the diamond discussed in appendix $C$. For exit the speed then drops to about 0.6 at $\tau=0.7$ before rising again and becoming much more Froudenumber dependent, presumably due to wave-like flow near the wedge. We do not understand these interesting results in any analytical way and it is not clear how to relate them to the present case of an accelerating exiting wedge, but the results suggest that some sort of model at $\tau=0$ might be possible.

\section{Conclusion}

The free-surface profile, pressures on the wetted body surface and body force have been computed for constant acceleration of exit for various wedge and box-shaped bodies. Free-surface profiles for the symmetric wedge were shown to converge to high accuracy and the effects of acceleration parameter were computed from initial submergence to almost complete exit. Corresponding results for asymmetric or truncated wedges, and for box-shaped bodies are less reliable, especially for the pressure profile near the sharp corners where the spatial derivative of the complex potential becomes large. This is to be expected since in reality, vortices will be shed at the corners. These would need to be included in any future models. Nevertheless, the results for the freesurface profiles may be accurate, since the free surface is distant from any shed vortices and so may provide valuable benchmark results for other numerical models and/or experiments. What is clear, even for the box, is that the numerical method agrees very well with the analytical added-mass model for small times and this simplified model indicates correct behaviour even for larger times. Given this agreement and its relative simplicity, the added mass model may therefore be of real use in engineering contexts.

\section{Appendix A. Mathematical formulation}

We describe the motion of the system of fluid particles and the moving body in a two-dimensional complex Cartesian coordinate system. The particles on the 
body surface and the free surface can be considered by a mixed Eulerian and Lagrangian description.

\section{Initial-boundary-value problem}

The initial boundary-value problem (IBVP) is formulated to study the motion executed by an object moving into and out of a fluid. We can derive mathematical governing equation for the problem based on a system of fluid and the object in the time-dependent domain $\Omega(t)$ as shown in Fig. A.15.

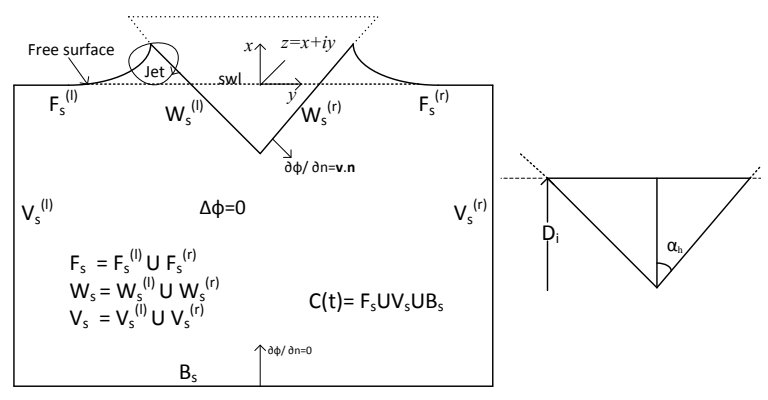

Figure A.15: Definition sketch for the domain representation of the fluid and the boundary.

\section{Governing equation}

Motion of the particles on the boundary of the domain as shown in Fig. A.15 is described by a mixed Lagrangian and Eulerian description. The domain of the problem is varying as the body and free surface moves. The particle position is represented with respect to its origin in the still water level (swl). Euler's field representation of velocity of the particles is given by the velocity field $\vec{V}_{z}=u(x, y, t) \widehat{i}+v(x, y, t) \widehat{j}$ where $u$ is the velocity component in $x$ direction and $v$ is the velocity component in $y$ direction and $\widehat{i}$ and $\widehat{j}$ are unit vectors along the directions $x$ and $y$ respectively.

Under the usual assumptions of incompressible and irritational flow, the fluid is governed by Laplace's equation in the simply connected domain $\Omega(t)$ at time $t$

$$
\nabla^{2} \phi(z, t)=0, \quad z=x+i y .
$$

We can compute the 2D stream function $\psi$, from the scalar velocity potential $\phi$, and vice versa using the Cauchy-Riemann equations

$$
\begin{aligned}
& u=\frac{\partial \phi}{\partial x}=\frac{\partial \psi}{\partial y} \\
& v=\frac{\partial \phi}{\partial y}=-\frac{\partial \psi}{\partial x}
\end{aligned}
$$

\section{Boundary conditions}

The boundary of the domain $\Omega(t)$ is represented by a simply-connected closed contour $C(t)$ as shown in Fig. A.16. The boundary $C(t)$ and domain $\Omega(t)$ are dependent on time due to the deformation of the free surface and displacement of the body. The $C(t)$ consists of three kinds of boundaries. The first part represents the boundary $C_{d}(t)$ where the mixed boundary conditions (dynamic and kinematic boundary conditions) are specified, while the other parts $C_{n}(t)$ and $C_{p}(t)$ describe the boundaries where Neuman boundary conditions (body and seabed boundary conditions) and distant periodic boundary conditions apply.

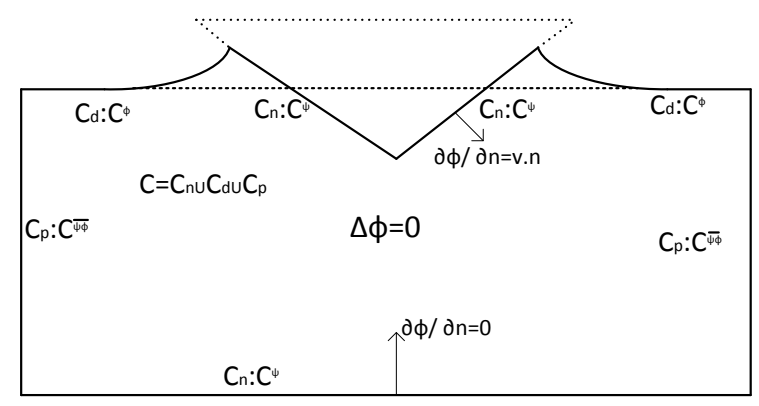

Figure A.16: Geometrical representation of the contour.

\section{Free-surface boundary conditions}

We impose the kinematic and dynamic boundary conditions on the free surface $F_{s}(t)$ which is a part of $C_{d}(t)$, see [10]. The kinematic free-surface boundary condition can be written in terms of material derivative as

$$
\frac{D(y-\eta(x, t))}{D t}=0
$$

The dynamic boundary condition on the free surface $F_{s}(t)$ can be obtained from the Bernoulli's equation by assuming that the pressure varies continuously across the free surface, i.e. there is no surface tension:

$$
\frac{\partial \phi(z, t)}{\partial t}+\frac{1}{2} \omega \bar{\omega}+g y+\frac{P_{a}}{\rho_{w}}=0, \quad \forall z \in F_{s}(t),
$$

where $P_{a}$ is the atmospheric pressure, $\rho_{w}$ is the density of the fluid, $\omega=u-i v$ is the complex velocity and $g$ is the gravitational acceleration. We can further assume that the pressure along the free surface is atmospheric which can be taken to be zero. We can rewrite the boundary condition for the representation in the $\mathrm{Eu}-$ lerian description as

$$
\frac{\partial \phi(z, t)}{\partial t}+\frac{1}{2} \omega \bar{\omega}+g y=0, \quad z \in F_{s}(t) .
$$




\section{Neumann boundary conditions}

We assume that the fluid particles along the wetted part $W_{s}(t)$ of the body can not cross into the body or move away from it, leaving a void. Thus the normal velocity of the flow around the submerged body is equal to the normal velocity of the body in contact with the fluid, i.e.

$$
\frac{\partial \phi(z, t)}{\partial n}=\phi_{n}=V_{z} \cdot n, \quad \forall z \in W_{s}(t) .
$$

\section{Initial conditions}

The free surface $F_{s}(t)$ is a part of the contour $C_{d}(t)$, whereas the wetted surface $W_{s}(t)$, and the bottom surface $B_{s}(t)$ are a part of the $C_{n}(t)$. The position vector, velocity and stream function are given along $W_{s}(t)$, while the initial position and velocity potential of the free surface particles are assumed to be known along $F_{s}(t)$ at a time $t=0$. The vertical surface $V_{s}(t)$ is a part of the $C_{p}$ where the stream function and the velocity potential are unknown, but these are computed using the periodicboundary condition (see $[14,15])$.

\section{Solution technique for the IBVP}

The formulated initial boundary-value problem (IBVP) is the Laplace equation in the velocity potential $\phi(z, t)$ and the stream function $\psi(z, t)$. The complex coordinate $z=x+i y$ and the region $R(t)$ is defined as a region comprising of both points on the boundary $C(t)$ and the domain $\Omega(t)$ at an instant time $t$. The analytical complex potential $\beta(z, t)$ can be defined as

$$
\beta(z, t)=\phi(z, t)+i \psi(z, t), \quad \forall z \in R(t) .
$$

We compute the solutions for the problem by timestepping forward the kinematic and dynamic boundary conditions. From the kinematic free-surface boundary condition, we have

$$
\frac{D z}{D t}=u+i v \equiv \bar{\omega}
$$

From the dynamic free-surface boundary condition, we have the Lagrangian description of $(A .5)$ as:

$$
\frac{D \phi}{D t}-\frac{1}{2} \omega \bar{\omega}+g y=0 .
$$

The velocity can be computed by differentiating the complex potential with respect to $z$. This gives

$$
\omega(z, t)=\frac{d \beta(z, t)}{d z}=u(z, t)-i v(z, t) .
$$

Cauchy's integral theorem is valid for the analytic function $\beta(z, t)$, so that for any $z_{0}$ outside of any closed contour $C(t)$ lying within the fluid region $R(t)$ we have

$$
\oint_{C(t)} \frac{\beta(z, t)}{z-z_{0}} d z=0
$$

Here the closed contour $C(t)$ consists of the free surface on which the velocity potential $\phi$ is assumed to be zero initially and denoted by $C^{\phi}$ where $\phi$ is known, the wetted part of the body on which the stream function $\psi$ can be computed using Eq. (A.4) and denoted by $C^{\psi}$ where $\psi$ is known and the vertical boundaries on which velocity potential $\phi$ and stream function $\psi$ are not known and denoted by $C^{\overline{\psi \phi}}$, see Fig. A.16. We now explain the techniques to compute the complex potential and its time derivative by formulating Eq. (A.11) as Fredholm's integral equation of second kind which, in general, has a solution.

Fig. A.17 shows a part of the contour $C$ consisting of two elements between $z_{k}$ and $z_{k-1}$, where $\alpha_{k}$ is an angle between the lines connecting the point $z_{k}$ from both sides of the points $z_{k+1}$ and $z_{k-1}$. Mathematically, but not numerically, this is $\pi$ for a smooth part of the surface.

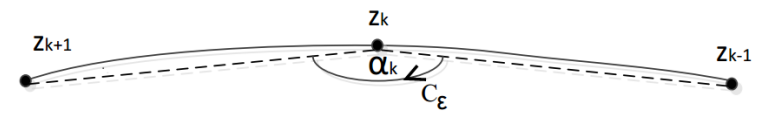

Figure A.17: Geometrical representation of the angle $\alpha_{k}$ for the numerical computation.

The closed contour $C$ includes the free surface $\left(F_{s}\right)$, the wetted surface of the body $\left(W_{s}\right)$, the bottom surface $\left(B_{s}\right)$ and the vertical surfaces $\left(V_{s}\right)$ of the domain. We assume that the point $z_{0}$ is initially outside the contour approaching the point $z_{k}$ on the contour.

We can now divide the contour integral of Eq. (A.11) into two parts $C_{k}$ and $C_{\epsilon}$, see Fig. A.15. By letting $z_{0}$ to approach from the outside of the contour to the point $z_{k}$, Eq. (A.11) can be written as

$$
\begin{aligned}
\oint_{C} \frac{\beta}{z-z_{0}} d z & =\oint_{C_{k}+C_{\epsilon}} \frac{\beta}{z-z_{k}} d z \\
& =\int_{C_{k}} \frac{\beta}{z-z_{k}} d z+\int_{C_{\epsilon}} \frac{\beta}{z-z_{k}} d z=0 .
\end{aligned}
$$

Using

$$
\lim _{\epsilon \rightarrow 0} \int_{C_{\epsilon}} \frac{\beta}{z-z_{k}} d z=i \alpha_{k} \beta\left(z_{k}\right),
$$


gives

$$
-i \oint_{C} \frac{\beta(z)}{z-z_{k}} d z=\alpha_{k} \beta\left(z_{k}\right)-i \int_{C_{k}} \frac{\beta(z)}{z-z_{k}} e^{i \theta} d s .
$$

We note that real or imaginary part of Eq. (A.14) can be equated to zero. However, this is chosen according to the point lying on the contour $C^{\phi}$ or $C^{\psi}$ and the solution of the integral equation formed by equating the real or imaginary part of Eq. (A.14) to zero, see [14,15].

To compute the complex potential, we need to know $\phi$ and $\psi$ which are known along $C^{\phi}$ and $C^{\psi}$ respectively. By considering that $z_{k}$ is on $C^{\psi}$ where $\phi$ is unknown and equating the real part of Eq. (A.14) to be zero, we get

$$
\begin{aligned}
& \operatorname{Re}\left\{-i \oint_{C} \frac{\beta(z)}{z-z_{k}} d z\right\} \\
= & \alpha_{k} \phi\left(z_{k}\right)-\operatorname{Re}\left\{i \int_{C_{k}}(\phi(s)+i \psi(s)) \frac{e^{i \theta}}{z-z_{k}} d s\right\} \\
= & \alpha_{k} \phi\left(z_{k}\right)-\operatorname{Re}\left\{\int_{C_{k}}(\phi(s)+i \psi(s)) \frac{i e^{i \theta}}{z-z_{k}} d s\right\} \\
= & \alpha_{k} \phi\left(z_{k}\right)-\int_{C_{k}} \operatorname{Re}\left\{\phi(s) \frac{i e^{i \theta}}{z-z_{k}}-i \psi(s) \frac{i e^{i \theta}}{z-z_{k}}\right\} d s \\
= & \alpha_{k} \phi\left(z_{k}\right)+\int_{C_{k}} \phi(s) \operatorname{Im}\left\{\frac{e^{i \theta}}{z-z_{k}}\right\} d s \\
& +\int_{C_{k}} \psi(s) \operatorname{Re}\left\{\frac{e^{i \theta}}{z-z_{k}}\right\} d s \\
= & 0,
\end{aligned}
$$

which we can write in the form

$$
\begin{aligned}
-\operatorname{Re}\left\{i \oint_{C} \frac{\beta(z)}{z-z_{k}} d z\right\}=\alpha_{k} \phi\left(z_{k}\right)+h\left(z_{k}\right) & \\
& +\int_{C_{k}} \phi(s) g\left(z_{k}, s\right) d s=0 .
\end{aligned}
$$

Similarly, by assuming that $z_{k}$ is on $C^{\phi}$ where $\psi$ is unknown and equating the imaginary part of Eq. (A.14) to be zero, we get

$$
\operatorname{Im}\left\{-i \oint_{C} \frac{\beta(z)}{z-z_{k}} d z\right\}
$$

$$
\begin{aligned}
& =\alpha_{k} \psi\left(z_{k}\right)+\operatorname{Im}\left\{-i \int_{C_{k}} \frac{\beta\left(z_{k}\right)}{z-z_{k}} e^{i \theta} d s\right\} \\
& =\alpha_{k} \psi\left(z_{k}\right)+\operatorname{Im}\left\{\int_{C_{k}} \frac{(\phi(s)+i \psi(s))}{z-z_{k}}-i e^{i \theta} d s\right\} \\
& =\alpha_{k} \psi\left(z_{k}\right)+\operatorname{Im}\left\{\int_{C_{k}} \frac{\phi(s)}{z-z_{k}}-i e^{i \theta} d s+\int_{C_{k}} \frac{\psi(s)}{z-z_{k}} e^{i \theta} d s\right\} \\
& =\alpha_{k} \psi\left(z_{k}\right)-\int_{C_{k}} \phi(s) \operatorname{Re}\left\{\frac{e^{i \theta}}{z-z_{k}}\right\} d s \\
& \quad+\int_{C_{k}} \psi(s) \operatorname{Im}\left\{\frac{e^{i \theta}}{z-z_{k}}\right\} d s \\
& =0,
\end{aligned}
$$

which can be written as

$$
\begin{array}{r}
\operatorname{Re}\left\{\oint_{C} \frac{\beta(z)}{z-z_{k}} d z\right\}=-\alpha_{k} \psi\left(z_{k}\right)+j\left(z_{k}\right) \\
-\int_{C_{k}} \psi(s) l\left(z_{k}, s\right) d s=0,
\end{array}
$$

where the functions $g h, j$ and $l$ are known. Eq. (A.15) and (A.16) are inhomogeneous Fredholm integral equations of the second kind, see [11]. We can also take the imaginary parts of Eq. (A.14) for the points on $\mathrm{C}^{\psi}$ and real part of Eq. (A.14) for the points on $C^{\phi}$, but it gives inhomogeneous Fredholm integral equations of first kind which do not have unique solutions (see $[14,15])$. Similarly for the time derivative we have

$$
\oint_{C} \frac{\frac{\partial \phi}{\partial t}+i \frac{\partial \psi}{\partial t}}{z-z_{0}} d z=0, \quad \forall z \in \Omega,
$$

giving

$$
\begin{aligned}
& \pi \frac{\partial \psi\left(z_{0}\right)}{\partial t}+\operatorname{Re}\left\{\int_{C_{0}} \frac{\frac{\partial \phi}{\partial t}+i \frac{\partial \psi}{\partial t}}{z-z_{0}} d z\right\}=0, \quad \forall z_{k} \in C^{\phi} \\
& \pi \frac{\partial \phi\left(z_{0}\right)}{\partial t}+\operatorname{Re}\left\{i \int_{C_{0}} \frac{\frac{\partial \phi}{\partial t}+i \frac{\partial \psi}{\partial t}}{z-z_{0}} d z\right\}=0, \quad \forall z_{k} \in C^{\psi}
\end{aligned}
$$

These equations are of the same form as Eqs. (1) and (2) and hence lead to identical equations to Eqs. (A.15) and (A.16) in the time derivative.

We now know the complex potential and its time derivative on the closed contour $C$. However, it can also be noted that we can compute the values for $\beta(z)$ and $\frac{\partial \beta(z)}{\partial t}$ inside the domain using the Cauchy's integral formula. The derivation can be given by

$$
\beta\left(z_{0}, t\right)=\frac{1}{2 \pi i} \oint_{C} \frac{\beta(z, t)}{z-z_{0}} d z,
$$


and differentiating with respect to $t$, we get

$$
\frac{\partial \beta\left(z_{0}, t\right)}{\partial t}=\frac{1}{2 \pi i} \oint_{C} \frac{\frac{\partial \beta(z, t)}{\partial t}}{z-z_{0}} d z .
$$

Higher-order derivatives can also be computed by extending the Cauchy's integral formula as

$$
f^{(n)}\left(z_{0}\right)=\frac{n !}{2 \pi i} \oint_{C} \frac{f(z)}{\left(z-z_{0}\right)^{(n+1)}} .
$$

This approach was taken by Cooker [3] and allows larger time steps to be taken. However, this would be very complicated to implement in the present case when the number of body points, and sometimes free-surface points, changes throughout time.

The complex velocity $\omega$ can be computed by differentiating the complex potential with respect to $z$ as

$$
\begin{aligned}
\omega\left(z_{0}, t\right) & =\frac{1}{2 \pi i} \oint_{C} \frac{\beta(z, t)}{\left(z-z_{0}\right)^{2}} d z, \\
\frac{\partial \omega\left(z_{0}, t\right)}{\partial t} & =\frac{1}{2 \pi i} \oint_{C} \frac{\frac{\partial \beta(z, t)}{\partial t}}{\left(z-z_{0}\right)^{2}} d z, \\
\frac{d \omega\left(z_{0}, t\right)}{d z} & =\frac{1}{\pi i} \oint_{C} \frac{\beta(z, t)}{\left(z-z_{0}\right)^{3}} d z .
\end{aligned}
$$

The pressure at the free surface is assumed to be zero and the pressure inside the contour can be found by a rearrangement of Bernoulli's equation as

$$
\frac{-P(x, y, t)}{\rho}=\frac{\partial \phi}{\partial t}+\frac{1}{2} \omega \bar{\omega}+g y .
$$

We can define the acceleration from the kinematic boundary condition as

$$
\frac{D \omega(z, t)}{D t}=a_{x}\left(r_{z}, t\right)+i a_{x}(z, t), \forall z \in C(t) .
$$

The acceleration inside the contour can be calculated using

$$
a_{x}(x, y, t)+i a_{y}(x, y, t)=\frac{\partial \omega}{\partial t}+\omega \cdot \frac{d \omega}{d t} .
$$

\section{Appendix B. Numerical formulation}

Here we explain a numerical formulation to compute the values based on the derivations given by Vinje and Brevig [14,15], and Barringer [2]. The collocation method is used to solve Eqs. (A.15), (A.16), (A.18) and (A.19). The nodal points are generated by dividing the contour $C$ into finite number of elements as shown in Fig. B.18. To generate points on the free surface and the body surface, we choose appropriate point-spacing

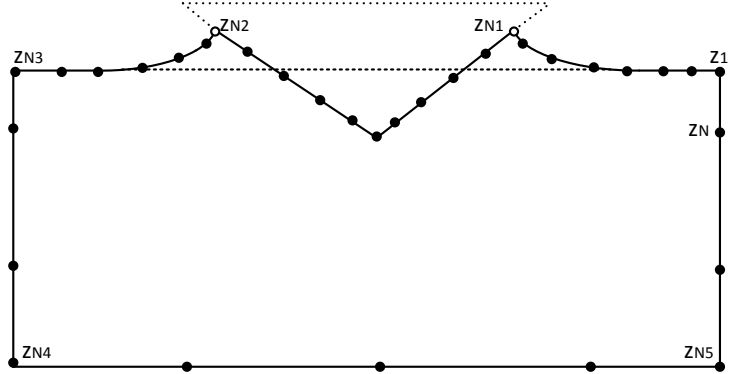

Figure B.18: Geometrical representation of the collocation points along the contour $\mathrm{C}$.

ratios by considering the fact that the points close to the body in motion are affected much more than points far from the body. We can also note that the point-spacing ratios and the time-step size play a very important role in the computation of the solution and need to be chosen carefully to compute the numerical results without breaking the computation and to achieve long runs of the code.

We know either the real or imaginary part of the complex potential $\beta(z)$ and its time derivative $\frac{\partial \beta}{\partial t}$ on every nodal point of the contour $C$. By assuming that the complex potential and its time derivative vary linearly in the complex variable $z$, we can write the complex potential for all $z$ on the contour $C$ as follows

$\beta(z)= \begin{cases}\frac{z-z_{j}}{z_{j-1}-z_{j}} \beta_{j-1}+\frac{z-z_{j-1}}{z_{j}-z_{j-1}} \beta_{j} & \forall z \in\left[z_{j-1}, z_{j}\right], \\ \frac{z-z_{j}}{z_{j+1}-z_{j}} \beta_{j+1}+\frac{z-z_{j+1}}{z_{j}-z_{j+1}} \beta_{j} & \forall z \in\left[z_{j}, z_{j+1}\right],\end{cases}$

where $z_{j-1}, z_{j}$ and $z_{j+1}$ are three consecutive nodes on the contour, and the complex potential $\beta_{j-1}, \beta_{j}$ and $\beta_{j+1}$ are known values on the points $z_{j-1}, z_{j}$ and $z_{j+1}$ respectively. Hence, the complex potential and its time derivative can be expressed as a linear combination of the complex potential at all nodes along the contour as follows

$$
\begin{gathered}
\beta(z)=\sum_{j} \wedge_{j} \beta_{j}, \\
\frac{\partial \beta(z)}{\partial t}=\sum_{j} \wedge_{j} \frac{\partial \beta_{j}}{\partial t},
\end{gathered}
$$


where $\wedge_{j}(z)$ is the influence function at node $z_{j}$ which can be computed by using

$$
\wedge_{j}(z)= \begin{cases}\frac{z-z_{j+1}}{z_{j}-z_{j+1}} & \forall z \in\left[z_{j}, z_{j+1}\right], \\ \frac{z-z_{j-1}}{z_{j}-z_{j-1}} & \forall z \in\left[z_{j-1}, z_{j}\right], \\ 0 & \text { otherwise. }\end{cases}
$$

Now, the contour integral can be numerically approximated by

$$
\begin{aligned}
\oint_{C} \frac{\beta(z)}{z-z_{k}} d z & =\oint_{C} \frac{\sum_{j} \wedge_{j} \beta_{j}}{z-z_{k}} d z \\
& =\sum_{j}\left(I_{k, j}^{1}+I_{k, j}^{2}\right) \beta_{j},
\end{aligned}
$$

where

$$
\begin{aligned}
I_{k, j}^{1} & =\int_{z_{j-1}}^{z_{j}} \frac{z-z_{j-1}}{z_{j}-z_{j-1}} \cdot \frac{1}{z-z_{k}} d z \\
& =\frac{1}{z_{j}-z_{j-1}}\left[z+z_{k} \ln \left(z-z_{k}\right)-z_{j-1} \ln \left(z-z_{k}\right)\right]_{z_{j-1}}^{z_{j}} \\
& =\frac{z_{k}-z_{j-1}}{z_{j}-z_{j-1}} \ln \left[\frac{z_{j-1}-z_{k}}{z_{j-1}-z_{k}}\right]+1, \\
I_{k, j}^{2} & =\int_{z_{j}}^{z_{j+1}} \frac{z-z_{j+1}}{z_{j}-z_{j+1}} \cdot \frac{1}{z-z_{k}} d z \\
& =\frac{1}{z_{j}-z_{j+1}}\left[z+z_{k} \ln \left(z-z_{k}\right)-z_{j+1} \ln \left(z-z_{k}\right)\right]_{z_{j}}^{z_{j+1}} \\
& =\frac{z_{k}-z_{j+1}}{z_{j}-z_{j+1}} \ln \left[\frac{z_{j+1}-z_{k}}{z_{j}-z_{k}}\right]-1 .
\end{aligned}
$$

The function $\Gamma_{k, j}$ denotes the sum of the terms $I_{k, j}^{1}$ and $I_{k, j}^{2}$ as

$$
\begin{aligned}
\Gamma_{k, j} & =I_{k, j}^{1}+I_{k, j}^{2} \\
& =\frac{z_{k}-z_{j-1}}{z_{j}-z_{j-1}} \ln \left[\frac{z_{j}-z_{k}}{z_{j-1}-z_{k}}\right]+\frac{z_{k}-z_{j+1}}{z_{j}-z_{j+1}} \ln \left[\frac{z_{j+1}-z_{k}}{z_{j}-z_{k}}\right] .
\end{aligned}
$$

However, we note that the $\lim _{z \rightarrow 0}\left(z \ln \frac{1}{z}\right)$ goes to zero. Thus to avoid the singularity, the function can be ex- plicitly expressed as

$$
\Gamma_{k, j}= \begin{cases}\frac{z_{j-1}-z_{j+1}}{z_{j}-z_{j+1}} \ln \frac{z_{j+1}-z_{j-1}}{z_{j}-z_{j-1}} & \text { for } k=j-1, \\ \frac{z_{j+1}-z_{j-1}}{z_{j}-z_{j-1}} \ln \frac{z_{j}-z_{j+1}}{z_{j-1}-z_{j+1}} & \text { for } k=j+1, \\ \ln \frac{z_{j+1}-z_{j}}{z_{j-1}-z_{j}} & \text { for } k=j .\end{cases}
$$

Finally, we note that the closed contour begins with the node $z_{1}$ and ends with the node $z_{N}$. We need to choose the values of $z_{j-1}=z_{0}$ at $\mathrm{j}=1$ and $z_{j+1}=z_{N+1}$ at $\mathrm{j}=\mathrm{N}+1$ when we evaluate the function $\Gamma_{k, j}$ which depends on the values of $z_{j-1}, z_{j}$ and $z_{j+1}$. The values are given by assuming a periodic domain and setting the index $j$ when $j=1 \Rightarrow j-1=N$ and when $j=N \Rightarrow j+1=1$. $N$ is the number of collocation points on the contour. Therefore, we can numerically compute the contour integral by expressing Eq. (A.11) as

$$
\oint_{C} \frac{\beta(z)}{z-z_{k}} d z \approx \sum_{j} \Gamma_{j}\left(z_{j-1}, z_{j}, z_{j+1}, z_{k}\right) \beta_{j},
$$

and similarly, the derivative of the contour integral can be written as

$$
\oint_{C} \frac{\frac{\partial \beta(z)}{\partial t}}{z-z_{k}} d z \approx \sum_{j} \Gamma_{j}\left(z_{j-1}, z_{j}, z_{j+1}, z_{k}\right) \frac{\partial \beta_{j}}{\partial t} .
$$

We know $\phi$ on the free surface which is the part of $C^{\phi}$ and take the real and imaginary parts according to Eqs.(A.15), (A.16), (A.21) and (A.22) while solving Eqs.(B.10) and Eq. (B.11).

Previous publications using this method were coded in Fortran. This has now been translated into Matlab which allows the easy use of built-in functions and better graphics.

\section{Appendix C. Added mass force for wedge}

In an unbounded fluid, particles near to a moving body are accelerated due to the motion executed by the body. The body experiences hydrostatic and hydrodynamic forces. The hydrodynamic forces can be thought of in terms of some amount of fluid mass that moves with the body as an added mass $\left(m_{a}\right)$. More correctly it characterises the kinetic energy in the fluid. The motion of the system of the fluid and body can be predicted using Lagrangian dynamics, see [2] for more 
details.

The model we use here takes advantage of the fact that at $t=0$, the flat free surface is a line of equipotential $\phi=0$. Thus for heave motion, the fluid flow is initially that in the lower half plane of a double body i.e. a diamond shape of the wedge and its reflection in the free surface, see [10]. This makes it possible to deduce the added masses of the various body shapes moving in heave. In the usual seakeeping theories, this added mass is simply the high-frequency limit of the radiation problem.

In this section, we derive the equation of the system of the fluid and body, and the analytical added-mass force for a symmetric wedge and a box body, as explained by Barringer [2]. Then the analytical force is compared with the force difference between the total numerical force computed using the nonlinear theory of Vinje and Bervig [14,15] and the buoyancy force, to verify that the forces agree at initial time $t=0$. The buoyancy force is simply that of the body below the still water line $y=0$, not the instantaneous water line which is not known a priori.

The equation for the motion of the system of water and the body consists of the hydrostatic force $\left(F_{b}\right.$, buoyancy force) and the hydrodynamic force $\left(F_{a}\right.$, added mass force). The buoyancy force is given by

$$
F_{b}=\rho_{w} g \forall(t),
$$

where $\rho_{w}$ is the fluid density, $g$ is gravitational acceleration, and $\forall(t)$ is submerged volume of the instantaneous portion of the body below the undisturbed free surface level at $y=0$ and a time $t$.

The added mass force can be defined from the energy of the system of the fluid and body. The kinetic energy is written as

$$
T=\frac{1}{2}\left(m_{a}+m_{b}\right) v^{2} .
$$

The potential energy can be given as

$$
V=\int_{0}^{y}\left(m_{b} g-\rho_{w} g \forall\right) d y=\left(m_{b} g-\rho_{w} g \forall\right) y .
$$

Thus the Lagrangian is,

$$
L=T-V=\frac{1}{2}\left(m_{a}+m_{b}\right) \dot{y}^{2}+\left(\rho_{w} g \forall-m_{b} g\right) y .
$$

Now we can apply the Lagrange's equation for the motion of the system in a single degree of freedom (vertical motion of the body):

$$
\frac{\partial L}{\partial y}-\frac{d}{d t}\left(\frac{\partial L}{\partial \dot{y}}\right)=0 .
$$

By using Eq.(C.4) and Eq.(C.5), we get

$$
\begin{aligned}
\frac{\partial L}{\partial y}-\frac{d}{d t}\left(\frac{\partial L}{\partial \dot{y}}\right) & =\frac{1}{2} \frac{d m_{a}}{d z} \dot{y}^{2}+\left(\rho_{w} g \forall-m_{b} g\right) \\
& -\frac{d}{d t}\left(\left(m_{a}+m_{b}\right) \dot{y}\right),
\end{aligned}
$$

and

$$
\begin{aligned}
\frac{d}{d t}\left(\left(m_{a}+m_{b}\right) \dot{y}\right) & =\left(m_{a}+m_{b}\right) \ddot{y}+\frac{d m_{a}}{d y} \frac{d y}{d t} \dot{y} \\
& =\left(m_{a}+m_{b}\right) \ddot{y}+\frac{d m_{a}}{d y} \dot{y}^{2} .
\end{aligned}
$$

We find

$$
\begin{aligned}
\frac{\partial L}{\partial y}-\frac{d}{d t}\left(\frac{\partial L}{\partial y}\right) & =-\frac{1}{2} \frac{d m_{a}}{d y} \dot{y}^{2}+\left(\rho_{w} g \forall-m_{b} g\right) \\
& -\left(m_{a}+m_{b}\right) \ddot{y}=0 .
\end{aligned}
$$

Hence the equation for the motion of the system is

$$
m_{b} \ddot{y}+m_{b} g-\rho_{w} g \forall=-m_{a} \ddot{y}-\frac{1}{2} \frac{d m_{a}}{d y} \dot{y}^{2},
$$

where the left-hand side is the equation of motion and the right-hand side is the added mass force $F_{a}$ which can be stated as

$$
F_{a}=-\frac{1}{2} \frac{d m_{a}}{d y} v^{2}-m_{a} \dot{v},
$$

where $m_{a}$ is the added mass, $y$ is the vertical axis, and $\dot{y}$ and $\ddot{y}$ are replaced by $v$ and $\dot{v}$ (the heave velocity and acceleration), respectively. This is exact, but one can not calculate the energy in the fluid easily except at time $t=$ 0 . In general the added mass would depend on the entire history of motion (i.e. memory), due to wave radiation. In a footnote, Wagner [16] gives the added mass for a symmetric wedge as

$$
m_{a}=\rho b^{2} G \tan \alpha,
$$

where the function $\mathrm{G}$ is given in terms of Gamma functions by

$$
G=\frac{\Gamma\left(1+\frac{\alpha}{\pi}\right) \Gamma\left(\frac{1}{2}-\frac{\alpha}{\pi}\right)}{\Gamma\left(1-\frac{\alpha}{\pi}\right) \Gamma\left(\frac{1}{2}+\frac{\alpha}{\pi}\right)}-1,
$$


and $b=D_{i}-y$ is the submerged depth of the wedge, where $D_{i}$ is initial draft and $y$ is change in draft. The $\Gamma(.$.$) are Gamma functions, see [1]. Wagner offers no$ explanation of this formula, so it is worthwhile giving a derivation here using the Schwarz-Christoffel mapping (Fig.C.19)
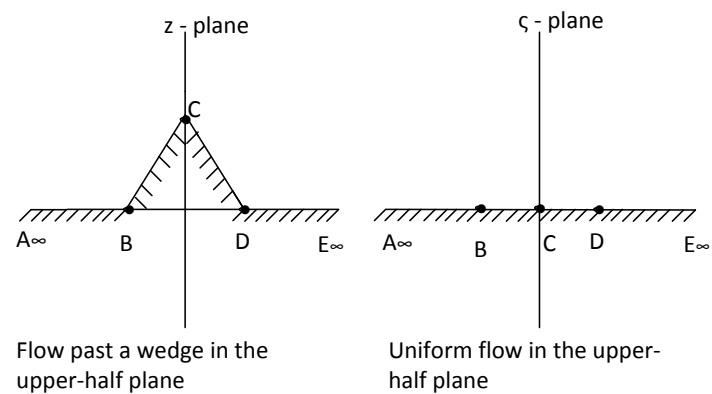

Figure C.19: Definition sketch for the flow mapping in the plane.

$$
\begin{aligned}
& z=K^{\prime} \int_{0}^{\zeta}(\zeta+1)^{\frac{\pi-\alpha}{\pi}-1}(\zeta)^{\frac{\pi+2 \alpha}{\pi}-1}(\zeta-1)^{\frac{\pi-\alpha}{\pi}-1} d \zeta+L \\
& z=K \int_{0}^{\zeta} \frac{(\zeta)^{\frac{2 \alpha}{\pi}}}{\left(1-\zeta^{2}\right)^{\frac{\alpha}{\pi}}} d \zeta+L .
\end{aligned}
$$

At point $\mathrm{C}: \zeta=0, z=a i \Rightarrow L=a i$.

At point D: $\zeta=1, z=b \Rightarrow b=K \int_{0}^{1} \frac{(\zeta)^{\frac{2 \alpha}{\pi}}}{\left(1-\zeta^{2}\right)^{\frac{\alpha}{\pi}}} d \zeta+a i$.

Using the identity,

$$
\frac{\Gamma(q) \Gamma(p)}{\Gamma(q+p)}=\int_{0}^{1} t^{q-1}(1-t)^{p-1} d t .
$$

see [1], and the substitution $\zeta=\sqrt{t}$, we can derive

$$
\begin{aligned}
& \qquad b=K \frac{\Gamma\left(\frac{\alpha}{\pi}-\frac{1}{2}\right) \Gamma\left(1-\frac{\alpha}{\pi}\right)}{\sqrt{\pi}}+a i \\
& \text { so that } K=\frac{(b-a i) \sqrt{\pi}}{\Gamma\left(\frac{\alpha}{\pi}-\frac{1}{2}\right) \Gamma\left(1-\frac{\alpha}{\pi}\right)} .
\end{aligned}
$$

We now set $K=|K| e^{-i a}$ where

$$
|K|=\frac{b}{\cos \alpha} \frac{\sqrt{\pi}}{\Gamma\left(\frac{\alpha}{\pi}-\frac{1}{2}\right) \Gamma\left(1-\frac{\alpha}{\pi}\right)} .
$$

So we can express

$$
\begin{aligned}
z & =|K| \int_{0}^{\zeta}\left(1-\frac{1}{\zeta^{2}}\right)^{-\frac{\alpha}{\pi}} d \zeta+a i \\
& =|K| \int_{1}^{\zeta}\left(1+\frac{\alpha}{\pi} \frac{1}{\zeta^{2}}+\cdots\right) d \zeta+b,
\end{aligned}
$$

since

$$
|K| \int_{0}^{1}\left(1-\frac{1}{\zeta^{2}}\right)^{-\frac{\alpha}{\pi}} d \zeta=b-a i
$$

Integrating gives

$$
z=|K|\left(\zeta-\frac{\alpha}{\pi} \frac{1}{\zeta}+\cdots\right)
$$

Thus by series reversion,

$$
\zeta=z^{\prime}+\frac{\alpha}{\pi} \frac{1}{z^{\prime}}+\cdots,
$$

where

$$
z^{\prime}=\frac{z}{|K|}
$$

We require $\beta(z) \rightarrow z$ as $z \rightarrow \infty$ for unit velocity at infinity. So

$$
\beta(z)=|K| \zeta=z+|K|^{2} \frac{\alpha}{\pi} \frac{1}{z}+\cdots .
$$

The added mass can be derived from the dipole coefficient as explained in [10]. So taking the coefficient of $1 / z$ we can conclude

$$
\left(\frac{m_{a}}{\rho}+\forall\right)=|K|^{2} \alpha=\frac{\left(a^{2}+b^{2}\right) \pi \alpha}{\left[\Gamma\left(\frac{\alpha}{\pi}-\frac{1}{2}\right) \Gamma\left(1-\frac{\alpha}{\pi}\right)\right]^{2}},
$$

where $\forall=b^{2} \tan \alpha$ is the body volume. Using further identities defined in [1] we obtain the equation

$$
\left(\frac{m_{a}}{\rho}+\forall\right)=b^{2} \tan \alpha \frac{\frac{\alpha}{\pi} \Gamma\left(\frac{\alpha}{\pi}\right) \Gamma\left(\frac{1}{2}-\frac{\alpha}{\pi}\right)}{\Gamma\left(\frac{\alpha}{\pi}+\frac{1}{2}\right) \Gamma\left(\frac{\alpha}{\pi}+\frac{1}{2}\right)} .
$$


With minor manipulation we finally obtain equations as stated by Wagner [16]. Fig. C.20 shows the relationship between the added mass, $m_{a}$ and the wedge half angle, $\alpha$. The added mass is non-dimensionalised in two different manners. On the left we non-dimensionalise with respect to the submerged body depth, $b$. Now we get

$$
\frac{m_{a}}{\rho \pi b^{2}}=\frac{\tan \alpha}{\pi} G
$$

On the right we non-dimensionalise with respect to body half width. Thus

$$
\frac{m_{a}}{\rho \pi a^{2}}=\frac{G}{\pi \tan \alpha}
$$

We note that the added mass for the wedge approaches

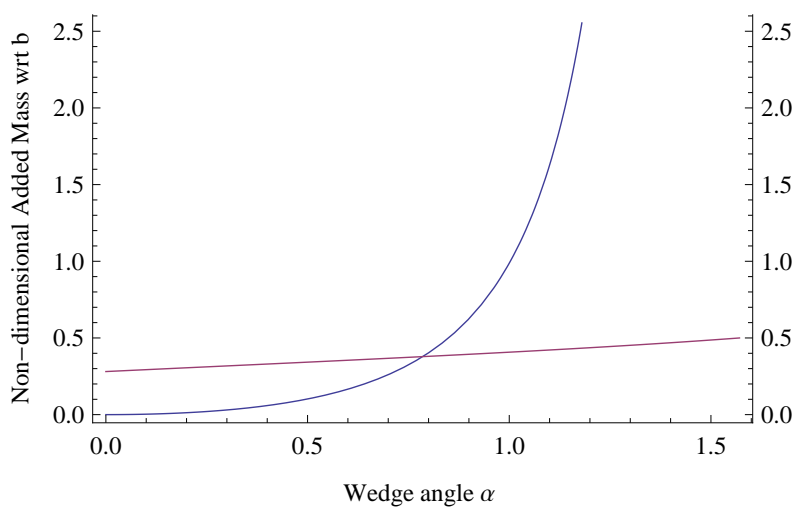

Figure C.20: Non-dimensional added mass (with respect to the depth $b$ and half width $a$ ) vs vertical half-wedge angle $\alpha$.

$\rho \pi a^{2} / 2$ as $\alpha$ approaches $90^{\circ}$. We expect this result since it is the added mass for a flat plate of half width $a$ (equivalent to the displaced mass of a cylinder radius $a$ ).

Differentiating the added mass with respect to $y$ and noting that $b$ is a function of $y$, we get

$$
\frac{d m_{a}}{d y}=-2 \rho b G \tan \alpha .
$$

Hence, by substitution in Eq.(C.10), we obtain the added mass force in a form suitable for comparison with the numerical calculations.

We compare the force difference $\left(F_{d}=F_{n}-F_{b}\right)$ with the analytic added mass force $\left(F_{a}\right)$ because the numerical force $\left(F_{n}\right)$ which we compute using the numerical method consists of the buoyant force $\left(F_{b}\right)$ and the force due to the pressure on the body (dynamic force), but the computation of the added-mass force does not include the buoyancy force. Figs. C.21 and (C.22) show the comparison of the computed force difference and the analytical added mass force computed for the symmetric wedge SW30 exiting with constant accelerations of 1 and 0.5 , respectively. It can be noted that both forces are very close to each other at time zero.

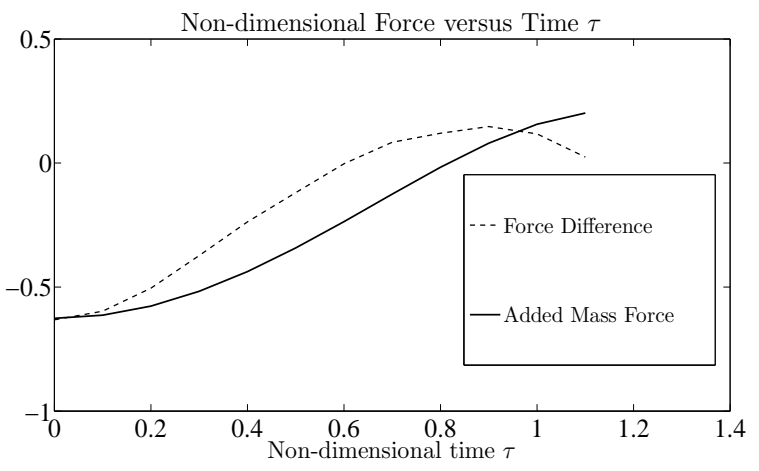

Figure C.21: Computed numerical force difference and added mass force showing added mass effect for the wedge SW30 exiting with constant acceleration $G_{\tau}=1$

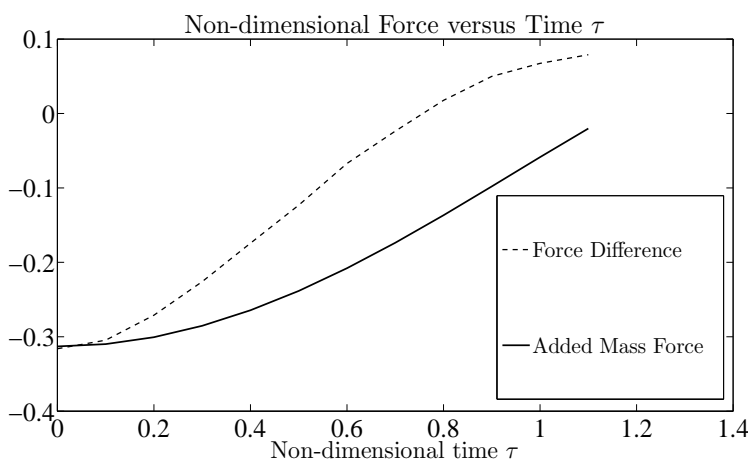

Figure C.22: Computed numerical force difference and added mass force showing added mass effect for the wedge SW30 exiting with constant acceleration $G_{\tau}=0.5$.

\section{Appendix D. Added mass force for box}

As for symmetric wedges, the added mass of the box body can also be found by conformal mapping, see [2]. Barringer shows that the added mass can be given in a similar way, but now involving complete elliptic integrals, as stated by Riabouchinski [13] but again without derivation. Thus for a box with corners $( \pm b, \pm a)$ moving 
in the $x$ direcction we have

$$
\frac{m_{a}}{\rho \pi a^{2}}=\frac{m}{[E(m)-(1-m) K(m)]^{2}}-\frac{4}{\pi} \frac{b}{a},
$$

where the parameter $m$ is related to the box dimensions by

$$
\frac{b}{a}=\frac{E(1-m)-m K(1-m)}{E(m)-(1-m) K(m)} .
$$

In our case the line $x=0,|y|>a$ represents the line of equipotential $\phi=0$. So the required added mass is simply half of that given by Eq. (D.1). Since these functions are less convenient that the gamma functions needed for the wedge and here there is only an implicit relation between the box aspect ratio and its added mass, Barringer fitted the results to an empirical formula:

$$
\begin{aligned}
& \frac{m_{a}}{\rho \pi a^{2}}=0.50589+0.26405 \sqrt{\frac{y}{a}} \\
& -0.00104839\left(\frac{y}{a}\right)^{\frac{3}{2}}-0.000014487\left(\frac{y}{a}\right)^{2}
\end{aligned}
$$

where $y=b$ is the draft of the box. The derivative of the added mass with respect to the draft $y$ is given by

$$
\begin{array}{r}
\frac{1}{\rho \pi a^{2}} \frac{d m_{a}}{d y}=0.132025 \frac{1}{\sqrt{a y}}+\frac{0.0251687}{a} \\
+0.001572585 \frac{y}{a}-0.000028974 \frac{y}{a^{2}}
\end{array}
$$

These are compared for the box body with the force difference computed numerically. Fig. D.23 shows the comparison of the computed force difference and the analytical added mass force computed for the box body $\mathrm{BX} 1$. It can be noted that both forces are fairly close to each other at time zero, but here we have resolution problems at the box corners as well as the intersection points.

\section{Acknowledgement}

We would like to express our thanks to Dr Jane Lawrie for her comments and the reviewers for their valuable suggestions to improve this paper.

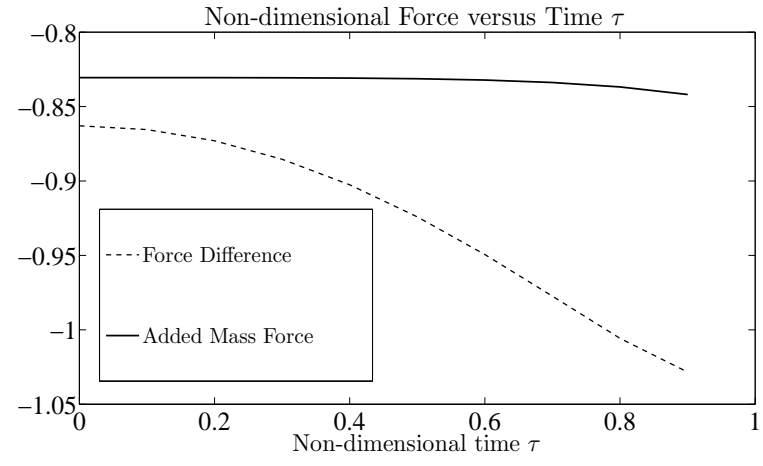

Figure D.23: Computed numerical force difference and added mass force showing added mass effect for the box BX1 exiting with constant acceleration $G_{\tau}=1$.

\section{References}

[1] Abramowitz M, Stegun IA. Handbook of mathematical functions, with formulas, graphs and mathematical tables. Dover Publication, Inc.; 1965, ISBN: 0-486-61272-4.

[2] Barringer I. Water exit of two-dimensional bodies [Ph.D. thesis]. UK: Brunel University; 1996.

[3] Cooker MJ. The interaction between steep water waves and coastal structures [PhD Thesis]. University of Bristol; 1990.

[4] DNV, Modelling and analysis of marine operations, 2011 http://www.stalforbund.com/_member/rp-h103_2011-04.pdf [access June 2014].

[5] Dobrovol'skaya ZN. On some problems of similarity flow of fluid with a free surface. J Fluid Mech 1969;36:805-29.

[6] Greenhow M. Wedge entry into initially calm water. Appl Ocean Res 1987; 9:259-75.

[7] Howison SD, Ockendon, JR, Wilson SK. Incompressible water-entry problems at small deadrise angles. J Fluid Mech 1991;222:215-30.

[8] Korobkin AA. A linearized model of water exit. J Fluid Mech 2013;737:368-86

[9] Longuet-Higgins MS, Cokelet ED. The deformation of steep surface waves on water I. A numerical method of computation. Proc. R Soc Lond 1976;A350:1-26.

[10] Newman JN. Marine Hydrodynamics. The MIT Press; 1977,ISBN 0-262-14026-8.

[11] Polyanin AD, Manzhirov AV. Handbook of integral equations. CRC Press LLC; 1998, ISBN 0-8493-2876-4.

[12] Rajavaheinthan R. Water exit of two-dimensional bodies [Ph.D. thesis]. UK: Brunel University; 2014.

[13] Riabouchinsk D. Sur la resistance des fluides. Strasbourg: Congress of Mathematics; 1920.p.568-85.

[14] Vinje T, Brevig P. Breaking waves on finite water depths, a numerical study. Ship Research Institute of Norway; 1981. R-111.81.

[15] Vinje T, Brevig P. Nonlinear two dimensional ship motion. Ship Research Institute of Norway; 1981. R-112.81.

[16] Wagner H. Uber Stoss und Gleitvorgänge an der Oberfläche von Flüssigkeiten. ZAMM 1932;12:193-215.

[17] Zhao R, Faltinsen O. Water entry of two-dimensional bodies. J Fluid Mech 1993;246:593-612. 